\title{
VvmiR160s/VvARFs interaction and their spatio-temporal expression/cleavage products during GA-induced grape parthenocarpy
}

Wenying Zhang ${ }^{1}$, Mostafa Abdelrahman²,3, Songtao Jiư ${ }^{4}$, Le Guan ${ }^{1}$, Jian Han ${ }^{1}$, Ting Zheng ${ }^{1}$, Haifeng Jia ${ }^{1}$, Changnian Song ${ }^{1}$, Jinggui Fang ${ }^{1}$ and Chen Wang ${ }^{1 *}$

\begin{abstract}
Background: Grape (Vitis vinifera) is highly sensitive to gibberellin (GA), which effectively induce grape parthenocarpy. Studies showed that miR160s and their target AUXIN RESPONSIVE FACTOR (ARF) responding hormones are indispensable for various aspects of plant growth and development, but their functions in GAinduced grape parthenocarpy remain elusive.

Results: In this study, the morphological changes during flower development in response to GA treatments were examined in the 'Rosario Bianco' cultivar. The precise sequences of VVmiR160a/b/c/d/e and their VVARF10/16/17 target genes were cloned, sequenced and characterized. The phylogenetic relationship and intron-exon structure of VVARFs and other ARF family members derived from different species were investigated. All VvmiR160s (except VVmiR160b) and VVARF10/16/17 had the common cis-elements responsive to GA, which support their function in GA-mediated grape parthenocarpy. The cleavage role of VvmiR160s-mediated VVARF10/16/17 was verified in grape flowers. Moreover, spatio-temporal expression analysis demonstrated that among VvmiR160 family, VvmiR160a/b/c highly expressed at late stage of flower/berry development, while VVARF10/16/17showed a reverse expression trend. Interestingly, GA exhibited a long-term effect through inducing the expression of VvmiR160a/b/c/e to increase their cleavage product accumulations from 5 to 9 days after treatment, but GA enhanced the expressions of VVARF10/16/ 17 only at short term. Pearson correlation analysis based on expression data revealed a negative correlation between VvmiR160a/b/c and VVARF10/16/17 in flowers not berries during GA-induced grape parthenocarpy.

Conclusions: This work demonstrated that the negative regulation of VVARF10/16/17 expression by VVmiR160a/b/C as key regulatory factors is critical for GA-mediated grape parthenocarpy, and provide significant implications for molecular breeding of high-quality seedless berry.
\end{abstract}

Keywords: VvmiR160s, VVARFs, Grape, Flower, Gibberellin, Parthenocarpy

\footnotetext{
* Correspondence: wangchen@njau.edu.cn

${ }^{1}$ College of Horticulture, Nanjing Agricultural University, Nanjing 210095,

China

Full list of author information is available at the end of the article
}

(c) The Author(s). 2019 Open Access This article is distributed under the terms of the Creative Commons Attribution 4.0 International License (http://creativecommons.org/licenses/by/4.0/), which permits unrestricted use, distribution, and reproduction in any medium, provided you give appropriate credit to the original author(s) and the source, provide a link to the Creative Commons license, and indicate if changes were made. The Creative Commons Public Domain Dedication waiver (http://creativecommons.org/publicdomain/zero/1.0/) applies to the data made available in this article, unless otherwise stated. 


\section{Background}

In angiosperms, flowering-time is a crucial part of the plant life cycle, providing a critical developmental switch from the vegetative growth to the reproductive stage to ensure seed production required for the survival of plant [1]. Floral development and flowering-time is regulated by a complex networks of genetic and epigenetic reprogramming [photoperiod, autonomous, vernalization/temperature, gibberellin (GA), and sucrose pathways] to ensure that flowering-time has coincided with suitable conditions for fertilization and seed dispersal [2-7]. This genetic and epigenetic reprogramming is attained by explicit control of the expression of key flowering genes at both transcriptional and post-transcriptional level in response to developmental and environmental stimuli $[3,4]$. In this context, microRNAs (miRNAs), a class of small, single-stranded, non-coding RNAs ranging from 19 to 25 nucleotides (nt) in length, have recently been identified as crucial regulators of gene expression through transcript cleavage and translational inhibition of target genes involved in the floral transition, floral patterning and development of floral organs [8-15]. However, the biological functions of these miRNAs have been characterized mainly in model plants $[16,17]$, while their roles during grapevine (Vitis vinifera) parthenocarpy and early ripening processes are still not fully understood.

Phytohormones, such as GA, are known to be essential for the regulation of grape flower development, berry expansion, ripening, and seedless berry induction, and the exogenous application of GA can trigger fruit parthenocarpy and early ripening in various grapevine cultivars $[15,18,19]$. Several reports demonstrated that different miRNA families (miR061, miR156, miR159, miR160, miR164, miR167, miR172, and miR319) and their target genes are implicated in GA signal during floral transition and fruit set development $[8,9,20]$. For instance, Arabidopsis AtmiR159 acts as a homeostatic regulator of GA signaling to direct the cleavage of genes encoding AtGAMYB proteins, causing a defect in anthers development and a subsequent reduction in floral fertility [21]. Similarly, auxin (AUX) is also known to be essential for fruit development and repining, and AUXIN RESPONSE FACTORs (ARFs) regulate the expression of a large set of auxin-responsive genes by binding to AUXIN RESPONSEELEMENTs (AuxREs) in their promoters [19]. Several studies in Arabidopsis and tomato plants demonstrated that miRNAs play significant roles in AUX signaling through controlling transcript abundance of ARF2/3/4/6/ 8/10/16 and 17 [22-24]. For example, Arabidopsis miR160a (AtmiR160a) mutant plants exhibited a significant reduction in AtmiR160a expression in floral organs in carpel (foc) inflorescences and irregular flower phenotype due to various embryonic defects [17]. Besides these, AtmiR160a mutant plants displayed up-regulation in ARF16 and ARF17 during embryo development in $f o c$ plants relative to wild-type plants, suggesting that AtmiR160a is essential for embryo development in Arabidopsis through negative regulation of $A R F$ genes [17]. Similarly, tomato (Solanum lycopersicum) SlmiR160 knocked down plants exhibited a perturbed ovary patterning and abnormal floral organ abscission, and the down-regulation of SlmiR160 was associated with the up-regulation of SIARF10A, SlARF1OB, and SIARF17, suggesting that SlmiRNA160 is needed for AUX-mediated floral and fruit development [24]. Although ARFs appear to have unique functions in some contexts, they also display overlapping functions among different plant species, for instance, a mutation in ARF8 resulted in the formation of seedless fruit in Arabidopsis and tomato plants [25, 26]. Therefore, characterization of $A R F$ members and their roles in floral development in different plant species are crucial.

Over the last decades, there has been a considerable increase in the economic importance of grape production for direct berry consumption and beverage production [27]. There are several reports demonstrated that the developmental process of grape flower affects berry fruiting type, yield and quality [28]. Therefore, the molecular regulatory mechanisms of grape flower development are essential to be determined. In our recent study, grapevine berries-treated with $\mathrm{GA}_{3}$ exhibited a significant up-regulation in several miRNA families in floral tissues, including miR159s and miR160s, suggesting a potential role of these miRNAs in GA signaling [29]. The potential role of VvmiR159c in GA signaling through GA-DELLA [SLENDER RICE 1 (SLR1)]VvmiR159c-VvGAMYB as a key molecular module for seedless grapevine development has recently been reported by our group [15]. However, the molecular mechanisms of miR160 and their specific target genes on modulating grape flower and parthenocarpy development remain elusive. In this context, we first examined the morphological changes in 'Rosario Bianco' cultivar during flower and berry development in response to $\mathrm{GA}_{3}$ treatment. Secondly, we isolated and cloned the precise sequences of $V v m i R 160 a / b / c / d / e$ in the floral tissues of grape cv. 'Rosario Bianco'. Thirdly, their ARF target genes were predicted by using $V v m i R 160 a / b / c / d / e$ sequences, together with updated grape mRNA database. The identified VvARFs were further characterized for their phylogenetic relationship and sequence homology with other ARFs derived from various plant species. The promoter motifs responsive to hormones including GA in VvMIR160s (VvmiR160 precursors) and VvARFs were analyzed, which was confirmed during our experiments. Finally, the cleavage role and cleavage sites of the different VvmiR160 members and their VvARF10/16/ 17target genes were verified in grape flowers by RNA-ligase-mediated Rapid Amplification of cDNA Ends (RLM-RACE) and Poly (A) polymerase -mediated 
3' rapid amplification of cDNA ends (PPM-RACE). In addition, the temporal-spatio expression and cleavage product accumulation of $V v m i R 160 a / b / c / d / e$ and VvARF10/16/17 target genes in response to GA application during grape development were investigated. Our results demonstrated that VvmiR160 modulates GA-mediated floral developmental and grapevine parthenocarpic regulation via its negative regulation of $V v A R F$ activity.

\section{Results}

Morphological changes of the floral and berry development during grapevine parthenocarpic processinduced by exogenous $\mathrm{GA}$ treatment

To investigate the effect of $\mathrm{GA}_{3}$ on grapevine floral development and parthenocarpy process, we compared the floral clusters and berries morphology of $\mathrm{GA}_{3}$-treated 'Rosario Bianco' grapevine cultivar relative to untreated control plants $(\mathrm{CK})$ at different time points $[0 \mathrm{~h}, 2 \mathrm{~h}, 1,5,9,10$, 45 and 95 days after treatment (0HAT, 2HAT, 1DAT, 5DAT, 9DAT, 10DAT, 45DAT and 95DAT respectively)] (Fig. 1a and b). Out of them, GA treatment was performed at 21 days after inflorescence, and the GA-treated plants reached anthesis at 9 DAT, while the control plants did not.
As shown in Fig. 1a and b, the $\mathrm{GA}_{3}$-treated plants exhibited a significant early anthesis and a remarkable increase in the length of spikes at 5DAT and 9DAT in comparison with untreated control plants (Fig. 1b). The $\mathrm{GA}_{3}$-treated inflorescences start opening on the 5DAT, while the control inflorescences remained closed (Fig. 1a). After peeling off buds for further observation, an obvious effect of $\mathrm{GA}_{3}$ treatment on 'Rosario Bianco' grapevine inflorescence was strongly observed on 5DAT and 9DAT with distinct floral morphology, including dark yellow and small anthers, long filaments, short styluses and big ovaries relative to untreated control plants (Fig. 1a). In addition, $\mathrm{GA}_{3}$-treated grapevine berries exhibited the long berry spikes and grains in contrast with control ones (Fig. 1b), and the high seedless ratio $(96.2 \%)$ relative to control plants $(\sim 5 \%)$ (Fig. 1a), suggesting that $\mathrm{GA}_{3}$ treatment possessed the significant effect in inducing 'Rosario Bianco' grapevine parthenocarpy.

Determination of the precise sequences of VvmiR160s in the floral tissues of grape cv. 'Rosario Bianco' by miRRACE

In this study, we employed miR-RACE technology to determine the precise sequences of VvmiR160 family

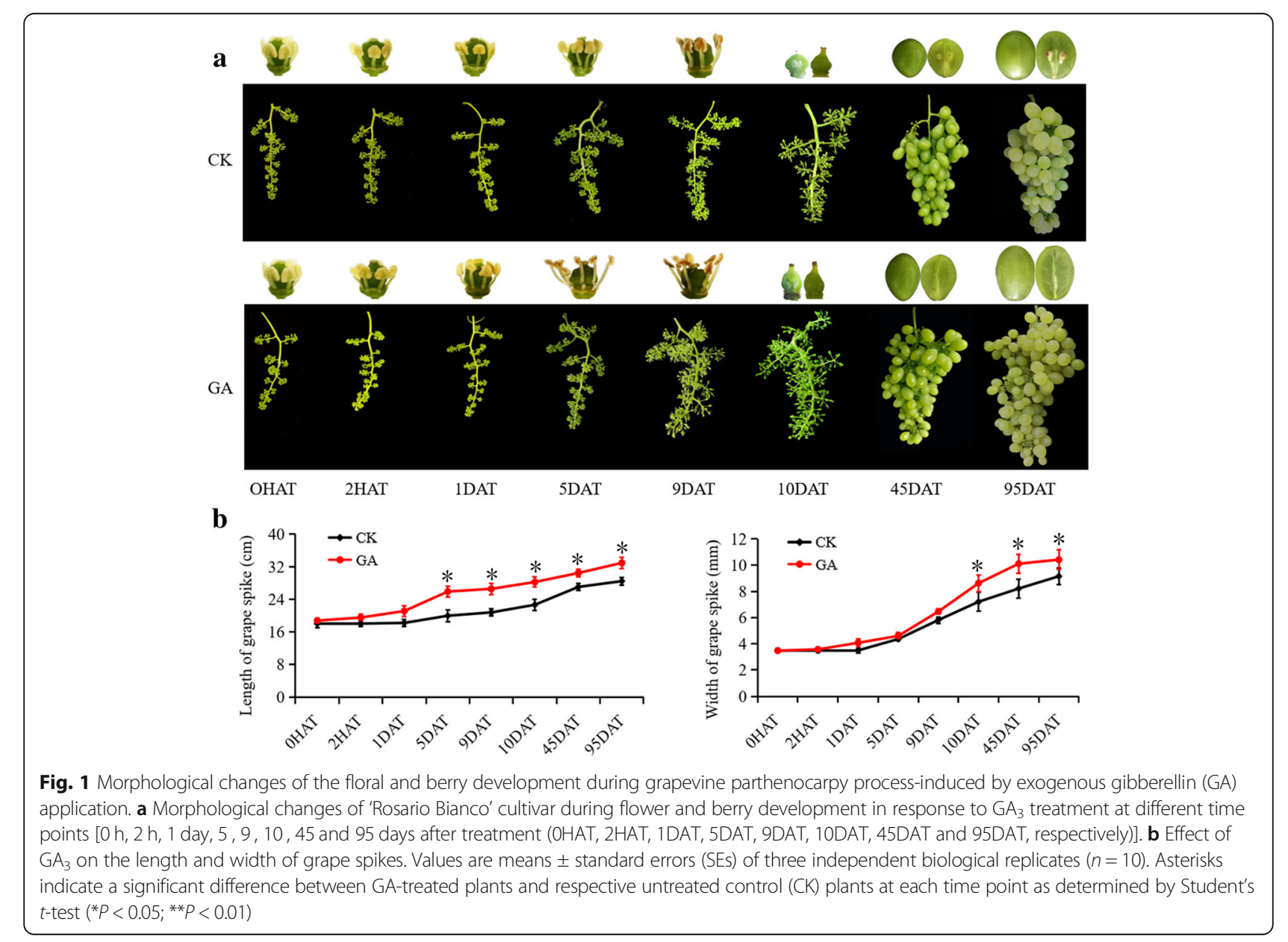


members in the floral tissues of grapevine cv. 'Rosario Bianco' (Fig. 2a and b). The PCR products of 3'- and 5'-miR-RACE for VvmiR160a/b/c/d/e members were 88 and 62 bp, respectively (Fig. 2a). All the miR-RACE PCR products were validated by subsequent cloning and sequencing. The sequence identity between our cloned sequences and validated miRNA in miRBase 21.0 was used to confirm the precision of the $3^{\prime}$ - and $5^{\prime}$-miR-RACE technique. VvmiR160a and VvmiR160b were identical both in length (21 nt) and nucleotide sequence "TGCCTGGCTCCCTGAATGCCA", with 14.2; 23.8; 23.8 and 38.0 percentage composition of A, T, G, and C, respectively (Fig. 2b). Whereas, VvmiR160c, VvmiR160d and VvmiR160e shared identical length and sequence
“TGCCTGGCTCCCTGTATGCCA", with 9,5; 28,5; 23,8 and 38,0 percentage composition of $\mathrm{A}, \mathrm{T}, \mathrm{G}$ and $\mathrm{C}$, respectively (Fig. 2b). The more GC content in the VvmiR160s is important for stabilization of the stem-loop hairpin structure. Both VvmiR160a/b and VvmiR160c/d/e exhibited high sequence similarity with one base variation $(\mathrm{A} / \mathrm{T})$ at the 15th site from the $5^{\prime}$-end (Fig. $2 \mathrm{~b}$ ). The primary transcripts of VvmiR160a/b/c/d/e (VvMIR160a-e) were cloned and sequenced, demonstrating that their lengths were about 501 bp (Fig. 2a), which were further used for secondary hairpin structure formation using Mfold online tool (http://unafold. rna.albany.edu/). These results confirmed the authenticity of $V_{v m i R} 160 a / b / c / d / e$, and all VvmiR160a/b/c/d/e members were located in the $5^{\prime}$-stem arm of their precursor's

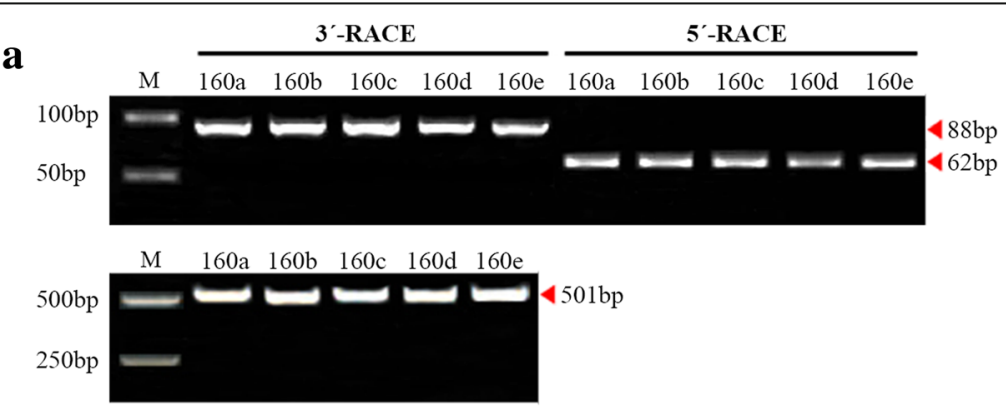

b

MiRBase sequences

Our cloned sequences

VvmiR160a TGCCTGGCTCCCTGAATGCCATC TGCCTGGCTCCCTGAATGCCA

VvmiR160b TGCCTGGCTCCCTGAATGCCATC TGCCTGGCTCCCTGAATGCCA

VvmiR160c TGCCTGGCTCCCTGTATGCCA TGCCTGGCTCCCTGTATGCCA

VvmiR160d TGCCTGGCTCCCTGTATGCCA TGCCTGGCTCCCTGTATGCCA

VvmiR160e TGCCTGGCTCCCTGTATGCCA TGCCTGGCTCCCTGTATGCCA
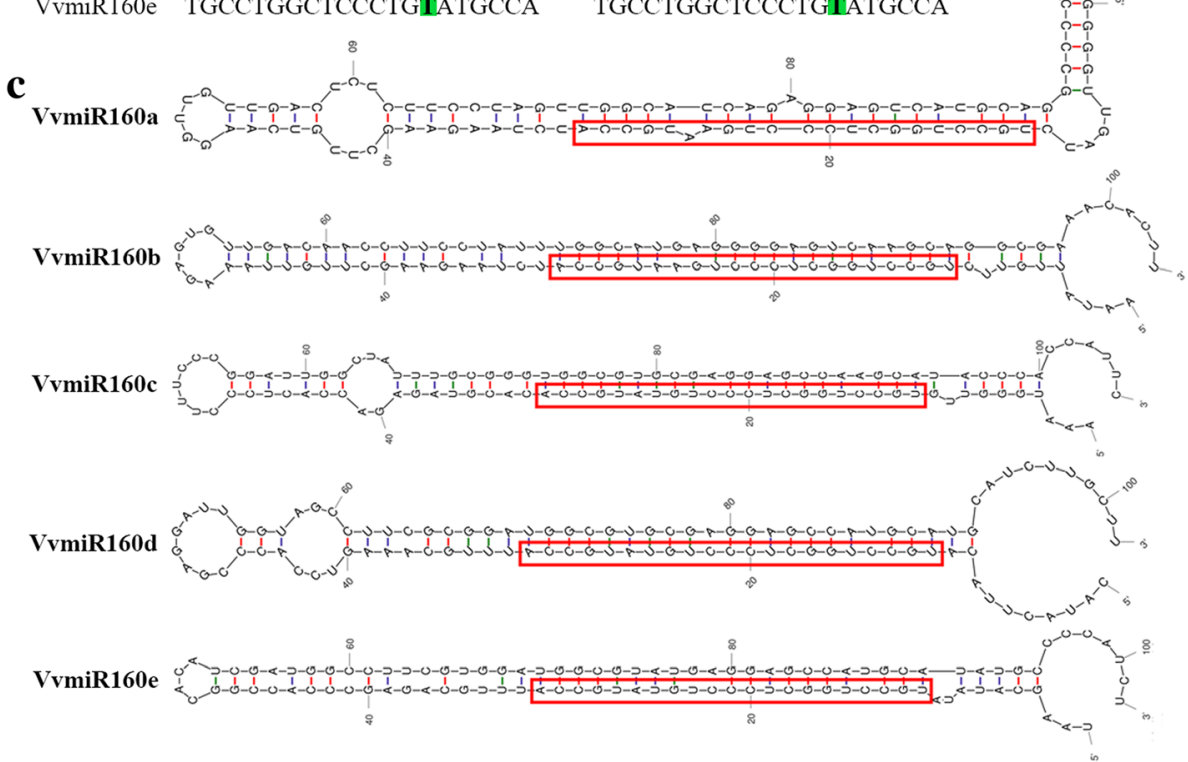

Fig. 2 Mature sequences of $\mathrm{V} v$ miR160s and the secondary structures of their precursors. a The PCR products of 3'-miR-RACE and 5'-miR-RACE for $V_{v m i R} 160 a / b / c / d / e$, respectively, and the cloning of their primary sequences. $\mathbf{b}$ Comparison of our cloned VvmiR160s precise sequences with the homologous sequences in grapes from MiRBase. $\mathbf{c}$ The secondary structures of VvmiR160s precursors, where red frames indicate the mature sequences of VvmiR160s at the 5'-end arms of their precursor structures 
loop-stem structures (Fig. 2c), suggesting that various members of VvmiR160 family possess a conserved position at their precursors.

\section{Identification, characterization and chromosomal distribution of $V v A R F$ genes}

Based on our identified sequences of VvmiR160s and the updated grape mRNA database (GRAPE_IGGP 12X assembly - v2 annotation), we employed the psRNATarget software (http://plantgrn.noble.org/psRNATarget/\#) to re-predict the specific target genes of VvmiR160s following the rules of target prediction as reported by Wang [30]. VvARF10/16/17 were identified as the candidate target genes of VvmiR160s (Additional file 1: Figure S1), and all target regions of VvmiR160s occurred in the coding sequence (CDS) of the corresponding VvARF target genes, and their interaction modes were cleaved (Table 1). Next, the complementary degree of $V v m i R 160: V v A R F$ pairs exhibited some divergence, for example,VvmiR160a/b and their VvARF16 target genes have three mismatch bases, while $V v m i R 160 c / d / e$ displayed only one mismatch base with VvARF16 (Fig. 3a), indicating some divergence in their cleavage roles. Also, high conservation at the sites with mismatch bases between VvmiR160s and VvARFs was observed (Fig. 3a), which might be one of the reasons for the conservation of target modes for VvmiR160s. All VvmiR160s and their $V v A R F$ target gene sequences were further mapped in grapevine chromosomes (Chrs) using MapInspect software (http://www.plantbreeding.wur.nl/ $\mathrm{uk} /$ software-mapinspect.html). All five VvmiR160s and three $V v A R F s$ identified in this study were distributed over four of the 19 grapevine chromosomes (Fig. 3b). Chr 10 contained two VvmiR160 members, whereas Chr 8, 12 and 13 contained only one VvmiR160 member (Fig. 3b). Mapping results demonstrated that VvmiR160a, VvmiR160d, and VvmiR160e are located on Chr 12, 8, 13 respectively, while both VvmiR160b and VvmiR160c were mapped into the same position on Chr 10 (Fig. 3b). In addition, VvARF10, VvARF16, and VvARF17 were mapped on Chr 8, 13 and 18, respectively (Fig. 3b). VvmiR160d:VvARF10 and VvmiR160e:VvARF16 pairs were located in the same position on Chr 8 and 13, respectively, whereas there is no co-localization of miR160s with VvARF17 on Chr 18 (Fig. $3 \mathrm{~b})$. Whether this chromosome neighboring of VvmiR160s and $V v A R F s$ might affect their cleavage activity, this needs further investigation.

Identification of $V_{V A R F s}$ and phylogenetic and diversified analyses of their orthologous sequences across diverse plant species

We cloned the three predicted $V v A R F 10 / 16 / 17$ gene sequences in 'Rosario Bianco' grapevine flowers, and their deduced amino acid sequences were identified (Additional file 2: Figure S2). The open reading frame (ORF) of VvARF10 was $2106 \mathrm{bp}$ encoding 701 amino acid residues, VvARF16 exhibited $2052 \mathrm{bp}$ encoding 683 amino acid residues, while $V v A R F 17$ possessed 1653 bp encoding 550 amino acid residues (Additional file 2: Figure S2). To study the phylogenetic relationships between the members of ARF family, an unrooted tree was constructed from an alignment of protein sequences of VvARF10/16/17 and their orthologous from different plant species by using Neighbour-joining method (Fig. 4a). Phylogenetic analysis reveals diversification and conservation of ARFs among different plant species (Fig. 4a). All the ARF family members fell into two major groups represented by 27 accessions (Fig. 4a). Group I contained all ARF10 and ARF16 classes, whereas group II contained all ARF17 class (Fig. 4a). The phylogenetic relationship between our VvARFs and orthologues one derived from NCBI demonstrated that VvARF16 has a close phylogenetic relationship with Arabidopsis (AtARF16), while VvARF10 and VvARF17 exhibited the closest relation to walnut (Juglans regia, JrARF10, and JrARF17, respectively), indicating a conserved nature of ARF family members among diverse plant species. On the other hand, VvARF10/16/17 have a distance phylogenetic relationship with cherry (Prunus avium, PaARFs) and peach (Prunus persica, PpARFs), indicating the diversification of evolution in diverse members of ARF protein family (Fig. 4a).

Analysis of the exon-intron structure of the three VvARF full-length cDNA sequences with the corresponding genomic DNA sequences showed that several members of $V v A R F$-CDS are disrupted by one to three introns (Fig. 4b). The maximum number of introns was detected in ARF10/16 members, while the lowest was detected in ARF17 (Fig. 4b). All the ARF members exhibited similar gene structures of intron position and length similar to their phylogenetic tree, which supports the reliability of the phylogenetic analysis (Fig. 4b). Three conserved domains (B3, AUX_rsep, and AUX_IAA) were identified, and their distribution in the proteins of respective ARFs in the phylogenetic tree has been presented (Fig. 4c). All the ARF proteins contained two well-conserved DNA binding domains B3 and Auxin_resp at the N-terminus (Fig. 4c). While VvARF16, SIARF10, JrARF10, and JrARF16 had additional AUX_IAA domain in the C-terminus (Fig. 4c), suggesting that ARFs have functional conservation in response to AUX signal in various plant species.

Next, motif composition analysis of ARFs proteins was carried out using the MEME 5.05 tool. The homologous ARFs with more close relationships from the phylogenetic tree possessed the similar motif compositions, and those with more far relationships had the conspicuous difference in their motif compositions (Fig. 4d), implying the more similar structures of homologous ARFs, the more conservation of their functions. Furthermore, the 
Table 1 Prediction of VvmiR160 target genes by bioinformatics

\begin{tabular}{|c|c|c|c|c|c|c|c|c|}
\hline MiR-Acc. & MiRNA mature sequence & $\begin{array}{l}\text { MiRNA } \\
\text { length }\end{array}$ & Target_Acc. & Expectation & UPE & Target regions & Inhibition & Multiplicity \\
\hline \multirow[t]{10}{*}{$\overline{V v m i R 160 a / b}$} & \multirow[t]{10}{*}{ TGCCTGGCTCCCTGAATGCCATC } & 23 & $\begin{array}{l}\text { VIT_218s0001g04180.1 } \\
\text { (VVARF17) }\end{array}$ & 1 & 20.766 & CDS:1755-1777 & Cleavage & 1 \\
\hline & & 23 & $\begin{array}{l}\text { VIT_208s0040g01810.1 } \\
\text { (VVARF10) }\end{array}$ & 1 & 23.188 & CDS:2389-2411 & Cleavage & 1 \\
\hline & & 23 & $\begin{array}{l}\text { VIT_208s0040g01810.3 } \\
\text { (VVARF10) }\end{array}$ & 1 & 23.188 & CDS:2513-2535 & Cleavage & 1 \\
\hline & & 23 & $\begin{array}{l}\text { VIT_208s0040g01810.2 } \\
\text { (VVARF10) }\end{array}$ & 1 & 23.188 & CDS:2389-2411 & Cleavage & 1 \\
\hline & & 23 & $\begin{array}{l}\text { VIT_208s0040g01810.4 } \\
\text { (VVARF10) }\end{array}$ & 1 & 23.188 & CDS:2534-2556 & Cleavage & 1 \\
\hline & & 23 & $\begin{array}{l}\text { VIT_218s0001g04180.2 } \\
\text { (VVARF17) }\end{array}$ & 1 & 20.766 & CDS:1755-1777 & Cleavage & 1 \\
\hline & & 23 & $\begin{array}{l}\text { VIT_213s0019g04380.1 } \\
\text { (VVARF16) }\end{array}$ & 1 & 22.721 & CDS:1566-1585 & Cleavage & 1 \\
\hline & & 23 & $\begin{array}{l}\text { VIT_213s0019g04380.3 } \\
\text { (VVARF16) }\end{array}$ & 1 & 22.721 & CDS:1566-1585 & Cleavage & 1 \\
\hline & & 23 & $\begin{array}{l}\text { VIT_213s0019g04380.4 } \\
\text { (VVARF16) }\end{array}$ & 1 & 22.721 & CDS:1701-1720 & Cleavage & 1 \\
\hline & & 23 & $\begin{array}{l}\text { VIT_213s0019g04380.2 } \\
\text { (VVARF16) }\end{array}$ & 1 & 22.721 & CDS:2147-2166 & Cleavage & 1 \\
\hline \multirow[t]{10}{*}{ VvmiR160c/d/e } & \multirow[t]{10}{*}{ TGCCTGGCTCCCTGTATGCCA } & 21 & $\begin{array}{l}\text { VIT_218s0001g04180.1 } \\
\text { (VVARF17) }\end{array}$ & 0.5 & 20.766 & CDS:1757-1777 & Cleavage & 1 \\
\hline & & 21 & $\begin{array}{l}\text { VIT_208s0040g01810.1 } \\
\text { (VVARF10) }\end{array}$ & 0.5 & 23.188 & CDS:2391-2411 & Cleavage & 1 \\
\hline & & 21 & $\begin{array}{l}\text { VIT_208s0040g01810.3 } \\
\text { (VVARF10) }\end{array}$ & 0.5 & 23.188 & CDS:2515-2535 & Cleavage & 1 \\
\hline & & 21 & $\begin{array}{l}\text { VIT_208s0040g01810.2 } \\
\text { (VVARF10) }\end{array}$ & 0.5 & 23.188 & CDS:2391-2411 & Cleavage & 1 \\
\hline & & 21 & $\begin{array}{l}\text { VIT_208s0040g01810.4 } \\
\text { (VVARF10) }\end{array}$ & 0.5 & 23.188 & CDS:2536-2556 & Cleavage & 1 \\
\hline & & 21 & $\begin{array}{l}\text { VIT_218s0001g04180.2 } \\
\text { (VVARF17) }\end{array}$ & 0.5 & 20.766 & CDS:1757-1777 & Cleavage & 1 \\
\hline & & 21 & $\begin{array}{l}\text { VIT_213s0019g04380.1 } \\
\text { (VVARF16) }\end{array}$ & 0 & 22.721 & CDS:1566-1585 & Cleavage & 1 \\
\hline & & 21 & $\begin{array}{l}\text { VIT_213s0019g04380.3 } \\
\text { (VVARF16) }\end{array}$ & 0 & 22.721 & CDS:1566-1585 & Cleavage & 1 \\
\hline & & 21 & $\begin{array}{l}\text { VIT_213s0019g04380.4 } \\
\text { (VVARF16) }\end{array}$ & 0 & 22.721 & CDS:1701-1720 & Cleavage & 1 \\
\hline & & 21 & $\begin{array}{l}\text { VIT_213s0019g04380.2 } \\
\text { (VVARF16) }\end{array}$ & 0 & 22.721 & CDS:2147-2166 & Cleavage & 1 \\
\hline
\end{tabular}

Note: The targeted information of VvmiR160s and VvARFs obtained by Pstarget software (http://plantgrn.noble.org/psRNATarget/\#)

parameters of protein physicochemical properties, including the number of amino acids, the molecular weight, the isoelectric point, the aliphatic index and the grand average of hydropathicity were analyzed (Additional file 3: Table S1). In contrast with the results in Fig. 4a and d, we revealed that although VvARF10 and JrARF10; VvARF16 and AtARF16; VvARF17 and JrARF17, had the close genetic distance in their gene sequences, their parameters in proteins had much more variation. This range of variability indicates that different
ARF proteins might operate in various microenvironments [31].

\section{Cis-element analysis of promoters of VvMIR160s and their VvARF target genes}

The promoter region is a critical factor for gene expression, and its sequence contains some essential cis-elements (functional components) which could reflect the potential functions of genes [32]. To recognize the possiblefunctions of $V v M I R 160 s$ and their $V v A R F s$ target 


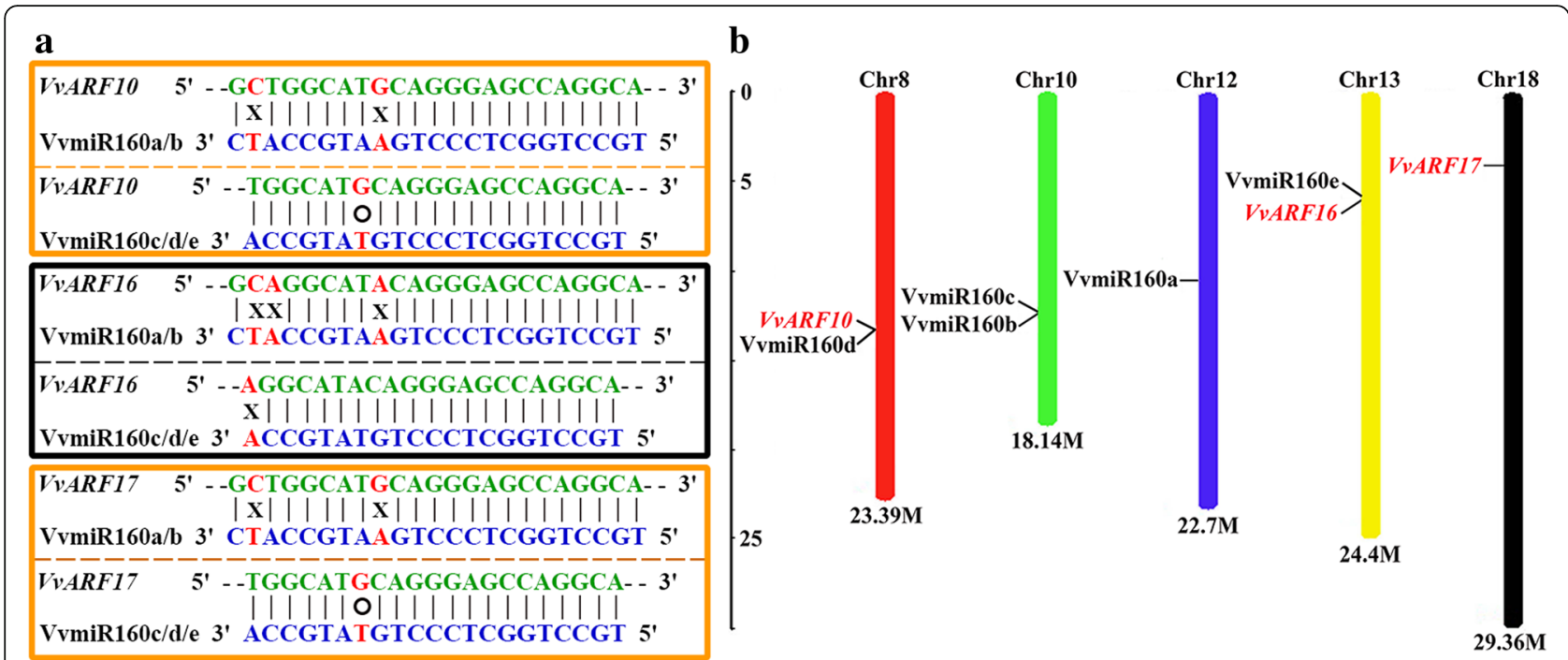

Fig. 3 Complementary degree and distribution of VvmiR160s and their VVARF targeted genes on grapevine chromosomes. a The complementary degree of VVmiR160s and their potential VVARF target genes. ' $X$ ' represents complementary mismatch, and ' $O$ ' represents the 0.5 mismatches. $\mathbf{b}$ The distribution of VVmiR160s and VVARFs on grapevine chromosomes. The chromosome numbers and sizes (Mb) are indicated at the top and bottom of each bar, respectively

genes in grapes, we analyzed the cis-elements in their promoters (Additional file 4: Table S2). All cis-elements were classified into five types, including light, hormone, tissue-specific, circadian and stress responsive elements (Fig. 5a). Light-related elements were the most abundant among all five types of motifs in the different VvmiR160 and VvARF members (Fig. 5a), which might be due to the important role of light in the photosynthesis process for plant growth and development. Whereas, the cis-elements responsive stress, hormone, and specific tissues development were also detected; however, their abundance was much lower than those of light responsive cis-element (Fig. 5a). Moreover, there were diverse in the type and number of promoters' cis-elements of VvMIR160s and their target VvARFs (Fig. 5a and Additional file 4: Table S2), indicating the diversification of various members from the same one family.

To further recognize the possible regulation mechanism of $V v m i R 160 s$ and their $V v A R F$ target genes in response to GA treatmentduring grape flower development, we scanned the hormone-related cis-elements in their promoter regions (Additional file 5: Table S3). Interestingly, we found that almost all VvmiR160 members (except VvmiR160b) and their VvARF10/16/17 target genes, had hormone responsive cis-elements, including indole acetic acid (IAA), GA, methyl jasmonate (MeJA), salicylic acid (SA), abscisic acid (ABA) and ethylene (ET) responsive cis-elements (Fig. 5b). However, both the composition and quantity of cis-elements related to hormones exhibited some variance among different VvmiR160 members and their VvARF target genes (Fig. $5 \mathrm{~b}$ and c), indicating that their potential functions might possess some unique as well as overlapping functions in grapes. Interestingly, all VvmiR160s (except VvmiR160b) and VvARFs had the common cis-elements responsive to GA and SA, which could be essential hormones for modulating grape flower and berry development. For example, VvmiR160a/c/d/e have cis-elements responsive to GA, among which, VvmiR160a exhibited the highest percentage (37\%), while VvmiR160c displayed the lowest (16\%). Similarly, VvmiR160d and VvmiR160e exhibited the highest percentage of cis-elements responsive to SA (50 and 33\%, respectively) in comparison with VvmiR160a and VvmiR160c (Fig. 5b). Our results supported the significant information for the prediction of the potential functions of VvmiR160:VvARF pairs in the modulation of grape growth and development through responding hormone signals.

\section{In vitro activity of $V v M I R 160 c$ and $V v A R F 10$ promoters in response to $\mathrm{GA}$}

A further analysis of the $V v M I R 160 c$ and $V v A R F 10$ promoters were conducted in order to confirm the activity of their promoters in response to GA. The VvMIR160c and VvARF10 promoter regions were fused to the $\beta$-glucuronidase (GUS) reporter gene after removal of $35 \mathrm{SCaMV}$ promoter in the binary vector pBI121, and the vector constructs are shown in Fig. 6a. The obtained constructs were transformed independently into tobacco plants using the Agrobacterium-mediated method. A pBI121-35S-GUS construct-containing $35 \mathrm{SCaMV}$ promoter served as positive control, while pBI101 construct-lacking 35SCaMV promoter served as negative control (Fig. $6 \mathrm{~b}$ ). To validate the presence of GA-related cis-elements in VvMIR160c and $V v A R F 10$ promoter regions, 0,30 and $50 \mu \mathrm{M}$ GA 


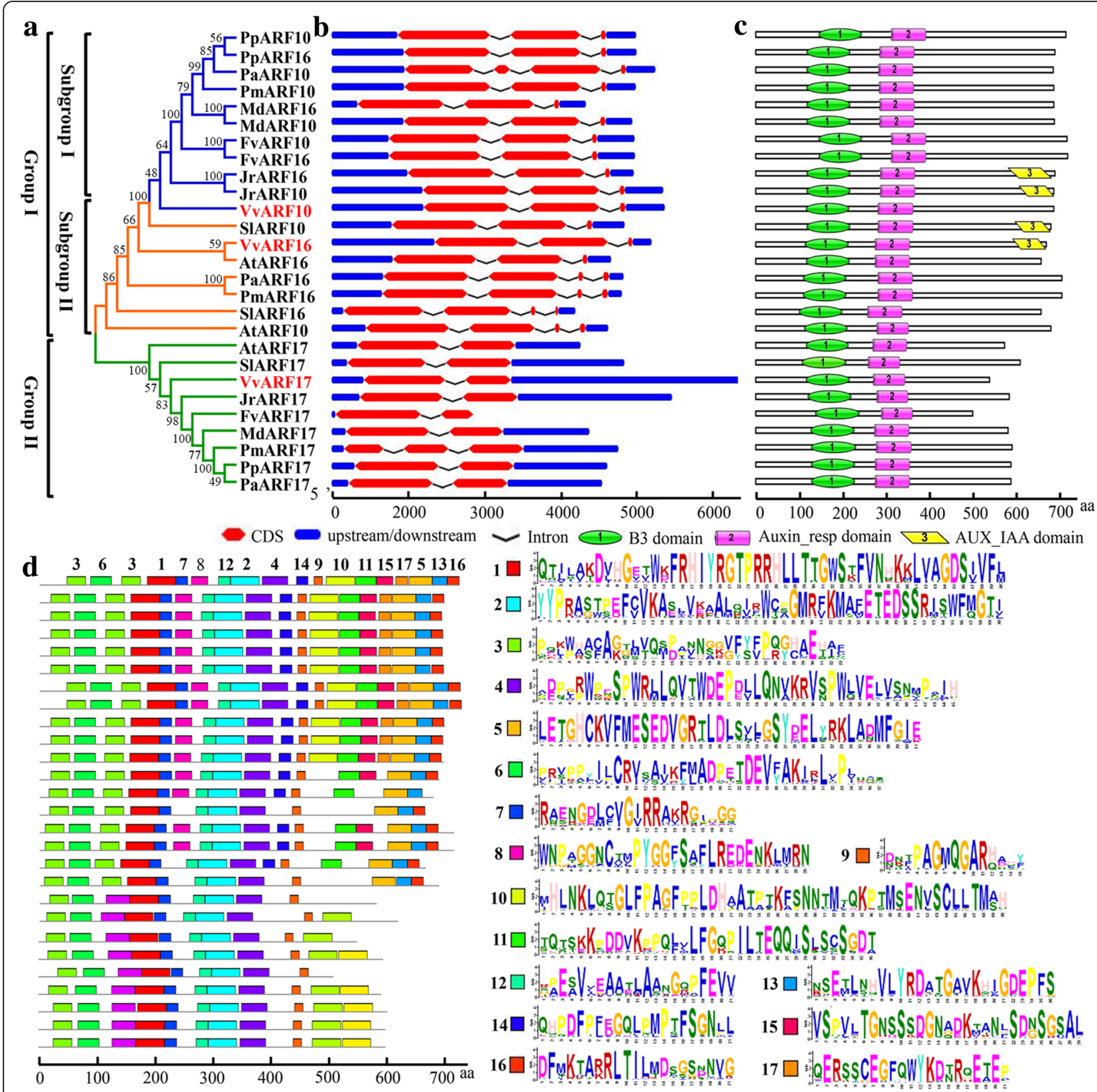

Fig. 4 The phylogenetic relationship, exon-intron organization, and conserved domain analyses among the grapevine ARF proteins and their orthologous sequences across different plant species. a The unrooted tree was generated using MEGA 7.0.21 program by neighbor-joining method. Bootstrap values from 1000 replicates are indicated at each branch. $\mathbf{b}$ The exon-intron composition of ARF genes. The coding (CDS) and up-/downstream regions are represented by red and blue boxes, respectively. Lines represent the introns. c Conserved domains in ARF proteins determined by searching the ARF protein sequences in NCBI Conserved Domain Database. B3: plant-specific B3-DNA binding domain, Auxin_resp: a conserved region of an auxin-responsive factor, AUX_IAA: C-terminal AUX_IAA domain. $\mathbf{d}$ The conserved motifs of ARF proteins in nine species were performed using the MEME program and arranged corresponding to the phylogenetic tree. Different motifs are highlighted with different color boxes and numbers. The length of boxes corresponded to motif length

were applied to the transgenic tobacco lines and the GUS activity was examined. The histochemical staining pattern revealed the effect of the GA treatments on the level of GUS activity in transformed tobacco leaves (Fig. 6b). As expected, the GUS gene driven by the $35 S C a M V$ promoter (positive control) was constitutively expressed in the transformed tobacco leaves under control and GA-treated conditions in comparison with negative control (pBI101 construct-lacking 35SCaMV promoter) transgenic lines (Fig. 6b and c). However, low level of GUS activity driven by the VvMIR160c and VvARF10 promoters 1 and 2 were observed in untreated $(0 \mu \mathrm{M} \mathrm{GA})$ 


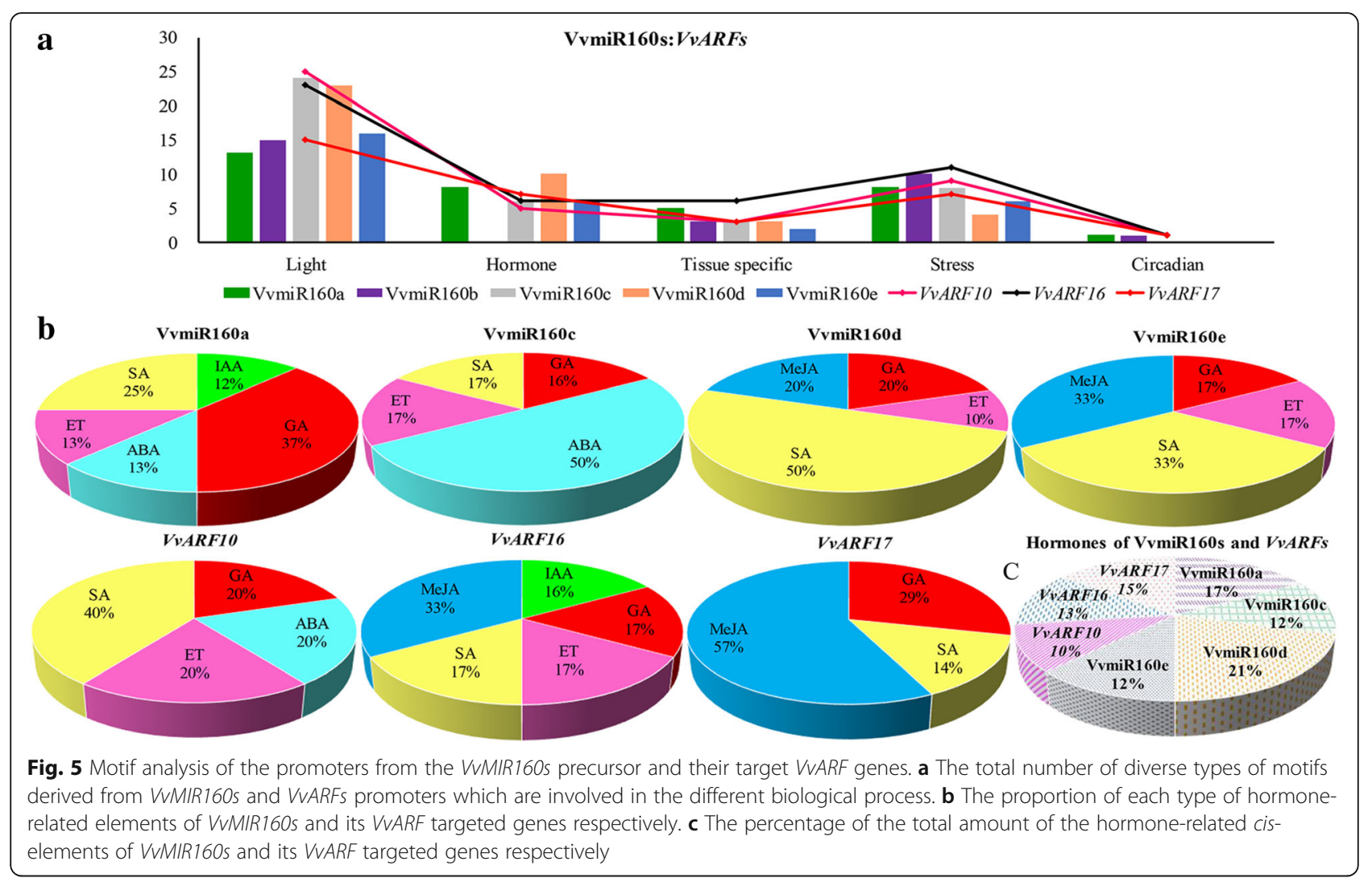

transformed tobacco leaves (Fig. 6b and c). GUS activity increased markedly in VvMIR160c and VvARF10 promoter 1-containing constructs (pBI121-p1VvmiR160c-GUS and pBI121-p1VvARF10-GUS) in the transgenic tobacco leaves-treated with 30 and $50 \mu \mathrm{M}$ GA, especially at higher dose $(50 \mu \mathrm{M}$ GA) in comparison with the untreated transgenic tobacco leaves (Fig. 6b and c). However, GUS activity almost did not changed in VvMIR160c and VvARF10 promoter 2-containing constructs (pBI121$p 2$ VvmiR160c-GUS and pBI121-p2VvARF10-GUS) in transgenic tobacco leaves treated with 30 and $50 \mu \mathrm{M}$ GA (Fig. $6 \mathrm{~b}$ and c). Our data clearly confirmed that $V v M I R 160 c$ and $V v A R F 10$ promoter 1 region with 1500 bp contained GA-related cis-responsive elements, which is essential for inducing GA response.

\section{Verification of VvmiR160-mediated VvARFs cleavage roles by RLM-RACE and PPM-RACE}

The interaction between VvmiR160 family members and VVARF target genes, and their regulatory mechanisms in grape floral tissues were investigated by our modified RLM-RACE and developed PPM-RACE procedures [30]. First, from RLM-RACE, the sequencing of the amplified 3'-end products revealed only one cleavage site in the middle of complementary regions of VvmiR160 family members and VvARF10/16/17 (Fig. 7). All the cleavage sites were mapped in the 9th site from the $5^{\prime}$ - end of VvmiR160a/b/c/d/e (Fig. 7), indicating the specificity of cleavage sites of miRNAs, which is consistent with previous studies [30, 33, 34]. Next, our developed PPM-RACE was employed to further confirm the target genes of VvmiR160s and their cleavage sites. The sequencing of the amplified $5{ }^{\prime}$-end products identified the same cleavage sites as those of the 3 '-end sequencing in the RLM-RACE experiment. In addition, the cleavage frequencies of 5 '-end product were less than those of $3{ }^{\prime}$-end product, which might be attributed to the more stability of 3 '-end fragments than 5 '-end one [30, 33, 34]. The consistent results of both the RLM-RACE and PPM-RACE experiments confirmed that VvARF10, $V v A R F 16$, and $V v A R F 17$ are the downstream target genes of $\mathrm{VvmiR} 160 \mathrm{a} / \mathrm{b} / \mathrm{c} / \mathrm{d} / \mathrm{e}$, and verified their cleavage interaction mode in grapevine inflorescence (Fig. 7). Although the cleavage sites of VvmiR160s were located on the same 9th position from their $5^{\prime}$-ends, these cleavage sites mapped on different positions in the respective $V v A R F$ target genes (Fig. 7). For instance, all cleavage sites of VvmiR160s were located at the 9th position from $5^{\prime}$-end of VvmiR160s, but located at the 1337th with $14 / 14$ from the $5^{\prime}$-end for VVARF10, 1304th with $16 / 16$ from the $5^{\prime}$-end for VvARF16 and the 1364th with $22 / 22$ from the $5^{\prime}$-end for VvARF17 (Fig. 7). 


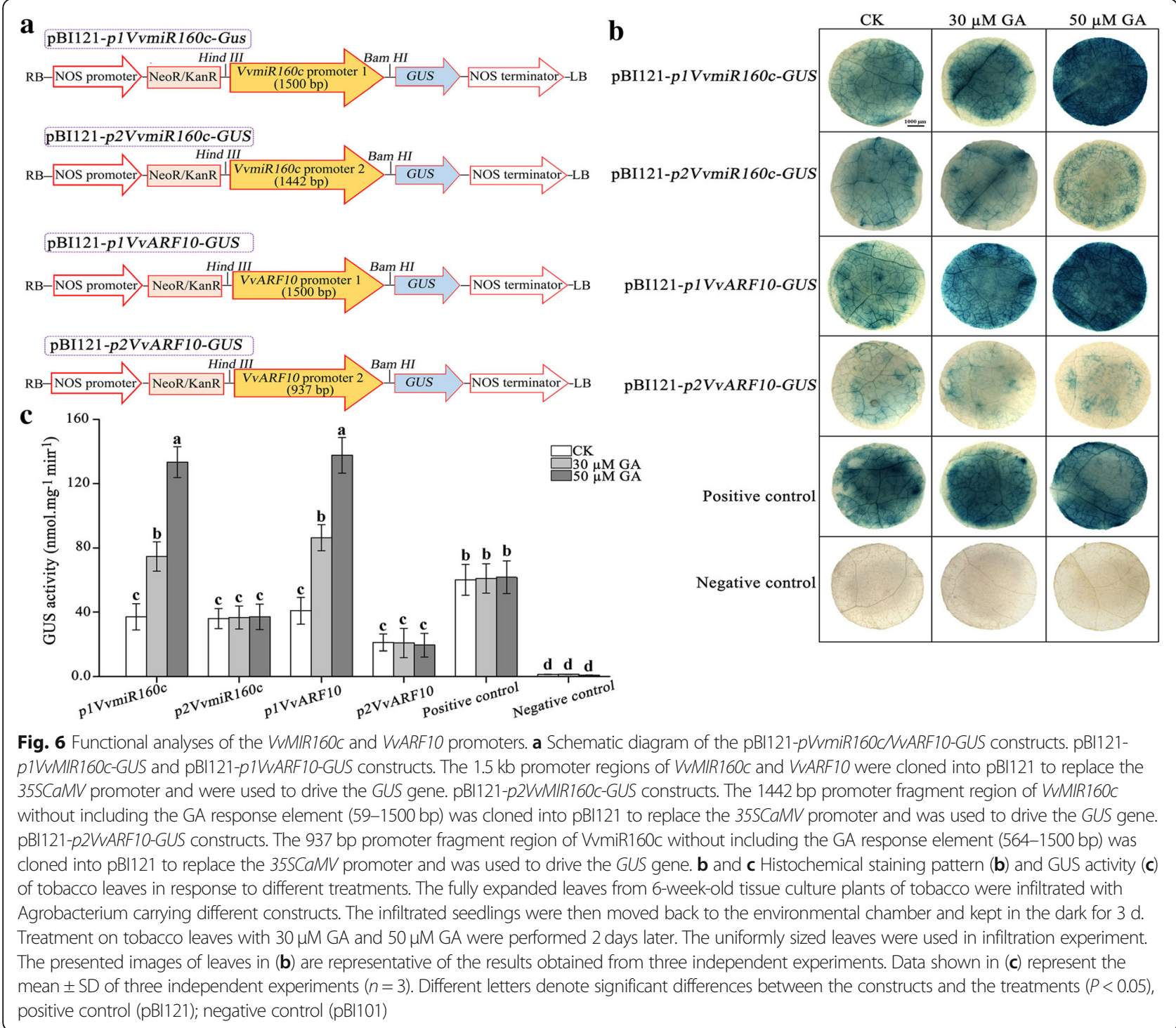

Differential expression profiles and correlation analysis of VvmiR160s and VvARFs during grape development

The effect of the interaction between $V v m i R 160 s$ and $V v A R F s$ during grape flower development was examined by measuring the spatio-temporal expression profiles of $V v m i R 160 s$ and $V v A R F s$ under control condition (Fig. 8a). As shown in Fig. 8a, the expression level of VvmiR160 family could be classified into three groups. In group one, $V v m i R 160 a / b / c$ exhibited the highest expression level during the late stage of floral development [from 22 days after inflorescence (22DAI) to 30DAI], and low expression during the early stage of floral development (Fig. 8a). In addition, VvmiR160a and $V v m i R 160 c$ exhibited similar expression trend with the highest expression level has been detected at 26DAI, while $V v m i R 160 b$ possessed the highest level at 30DAI (Fig. 8a). In group two, VvmiR160d exhibited a reverse expression trend relative to $V v m i R 160 a / b / c$, with high expression level has been detected at early floral development, followed by a gradual decrease till the late phase of floral development (Fig. 8a). In group three, $V v m i R 160 e$ showed a low expression level at all stages, suggesting that $V v m i R 160 e$ might have a slight role during grape floral development (Fig. 8a). Subsequently, the expression profiles of $V v m i R 160 a / b / c$ exhibited one upward trend along with berry development [from 1 day after anthesis (1DAA) to 86DAA], and reached the high peak till maturation (86DAA); while VvmiR160d exhibited a reverse expression trend relative to $V v m i R 160 a / b / c$, and $V v m i R 160 e$ showed a low expression level at all stages, suggesting that VvmiR160e might also have a slight role during grape berry development (Fig. 8b).

All three $V v A R F 10 / 16 / 17$ target genes exhibited a reverse expression trend in comparison with $V v m i R 160 a /$ 


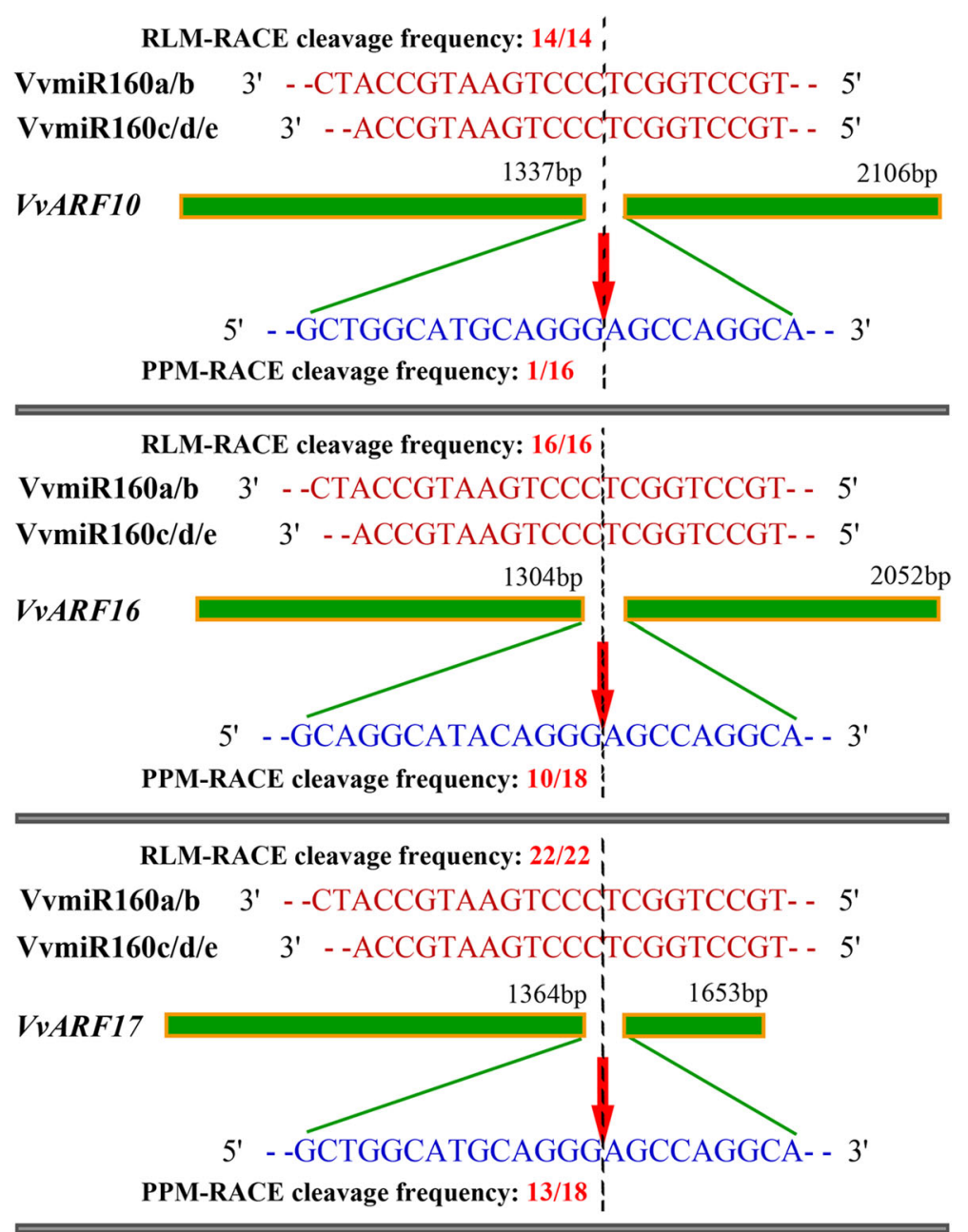

Fig. 7 Mapping of the VvmiR160s-mediated cleavage sites on VVARFs by PPM-RACE and RLM-RACE. The red arrows indicate the cleavage sites of VVmiR160s on VVARFs identified by 5' and 3'-ends of mRNA fragments cloned by PPM-RACE and RLM-RACE, respectively. The red and blue sequences represent the mature sequences of VVmiR160s and their target region sequences in VVARFs, respectively. Green strip denotes the remaining sequence outside the targeted region

$b / c$, where their expression levels increased during the early stage of flower/berry development and decreased gradually till the later stage (Fig. $8 \mathrm{a}$ and b). However, $V v A R F 10 / 16 / 17$ expression profiles were similar to $V v m i R 160 d$ expression during the different stages of grape flower/berry development (Fig. 8a and b). The Pearson correlation coefficient showed that VvARF10/ 16/17 expression exhibited a highly negative correlations $(r=-0.83$ to -0.94 for flowers; $r=-0.81$ to -1 for berries) with $V v m i R 160 a / b / c$ and positive correlation ( $r=0.93$ to 0.99 for flowers; $r=0.8842$ to 1 for berries) with $V v m i R 160 d$ (Fig. 8a and b). In general, the spatio-temporal expression and correlation analyses indicated that VvmiR160a/b/c were the key regulatory factors in grape floral and berry development through the negative regulation of $V v A R F$ expression.
The expression profiles of VvmiR160s and VvARFs in response to GA treatment during grape development GA is an essential factor for plant growth and development and the exogenous application of GA induced the expression level of VvmiR160s in plants [19, 29]. However, the relative effect of GA-mediated grape flower and berry development through $m i R 160 s$ remains elusive. In the present study, a comparative expression level of VvmiR160s and VvARF target genes in GA-treated and untreated control (CK) plants during floral development was carried out (Fig. 9a). The exogenous application of GA significantly up-regulated $V v m i R 160 a / b / c / e$ expression at 5DAT and 9DAT in comparison with untreated control plants (Fig. 9a). However, there was no significant effect of GA treatment on VvmiR160d expression (Fig. 9a). These results indicated that GA is a positive 

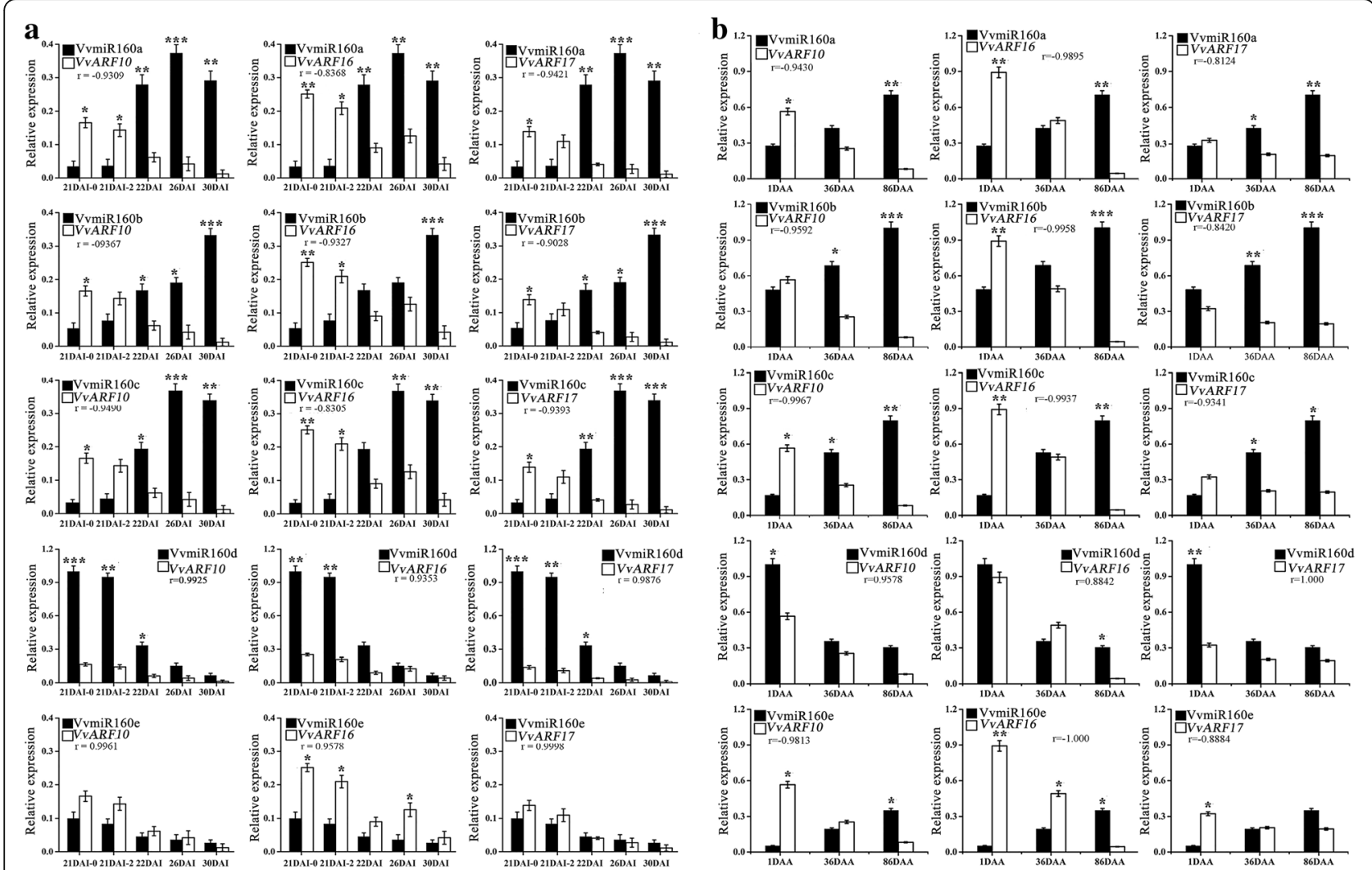

Fig. 8 Characterization of VVmiR160:VVARF expression and Pearson correlation analysis during grape flower and berry development. a Characterization of VvmiR160:VVARF expression and Pearson correlation analysis during grape flower development. $\mathbf{b}$ Characterization of VvmiR160:VVARF expression and Pearson correlation analysis during grape berry development. The relative expression of $V v m i R 160 \mathrm{a} / \mathrm{b} / \mathrm{c} / \mathrm{d} / \mathrm{e}$ and the three $\mathrm{VVARF10/16/17}$ target genes at different stages [21 days after inflorescences (21DAl), 22DAl, 26DAl and 30DAl; 1 day after anthesis (1DAA), 36DAA, 86DAA] of floral and berry development. Pearson correlation coefficient $(r)$ between VVmiR160 and VVARF expression are indicated. Each experiment was repeated three times. ANOVA test was used to identify significant differences, Asterisks indicated statistically significant differences at $\left({ }^{*} P<0.05 ;{ }^{*} P<0.01 ;{ }^{* * *} P<0.001\right)$ as determined by Student's t-test

long-term regulator of $\mathrm{VvmiR} 160 \mathrm{a} / \mathrm{b} / \mathrm{c} / \mathrm{e}$ during grape floral development, and the various members of miR160 family possessed diverse modes of response to GA. On the other hand, GA treatment induced VvARF10/16/17 expression mainly at $2 \mathrm{HAT}$ (Fig. 9a), implying that these target genes displayed a short-term response to GA treatment during floral development. Unlike flower development, GA treatment almost significantly up-regulated the expression of VvmiR160s and three VvARFs genes at 45DAT (45 days after treatment; berry expansion) (Fig. 9b). In addition, the three VvARFs were slightly up-regulated at their expression levels by GA at the maturation stage (Fig. 9b).

Further, we compared the expression levels of VvmiR160s and their VvARF target genes under GA treatments, we revealed that in grape flowers, VvmiR160a/c and $V v A R F 10 / 16 / 17$ expression exhibited some negative correlations $(r=-0.44$ to -0.53$)$ in response to the shortand long-term effect of GA treatment in comparison with untreated controls (Fig. 9c); however, in berries, their expression did not possess the negative correlation (Fig. 9d).
The correlation analysis indicated that GA might strength the regulatory roles of VvmiR160a/c on VvARF10/16/17 during floral development. However, VvmiR160b and its targets had negative correlations only in the short-term during floral development, while VvmiR160e and its targets possessed apparent negative correlations in the long-term. GA had no effect on VvmiR160d expression, compared with untreated control plants, and both VvmiR160d and VvmiR10/16/17 exhibited some positive correlation ( $r=0.56$ to 0.64 ) (Fig. 9c). In grape berries, VvmiR160a/b/c/d and VvARF10/16/17 expression exhibited some positive correlations $(r=0.69$ to 0.99$)$ in response to GA (Fig. 9d). These results suggested that VvmiR160a/b/c/d displayed various modes in responsive to GA during grape floral and berry development.

Considering the redundancy and complementary traits of miRNA's function, the correlation analysis of the total expression levels of all members of VvmiR160 family and each of $V v A R F$ target genes was investigated (Fig. 10a and b). Results showed that the total expression of VvmiR160s had negative correlations with 


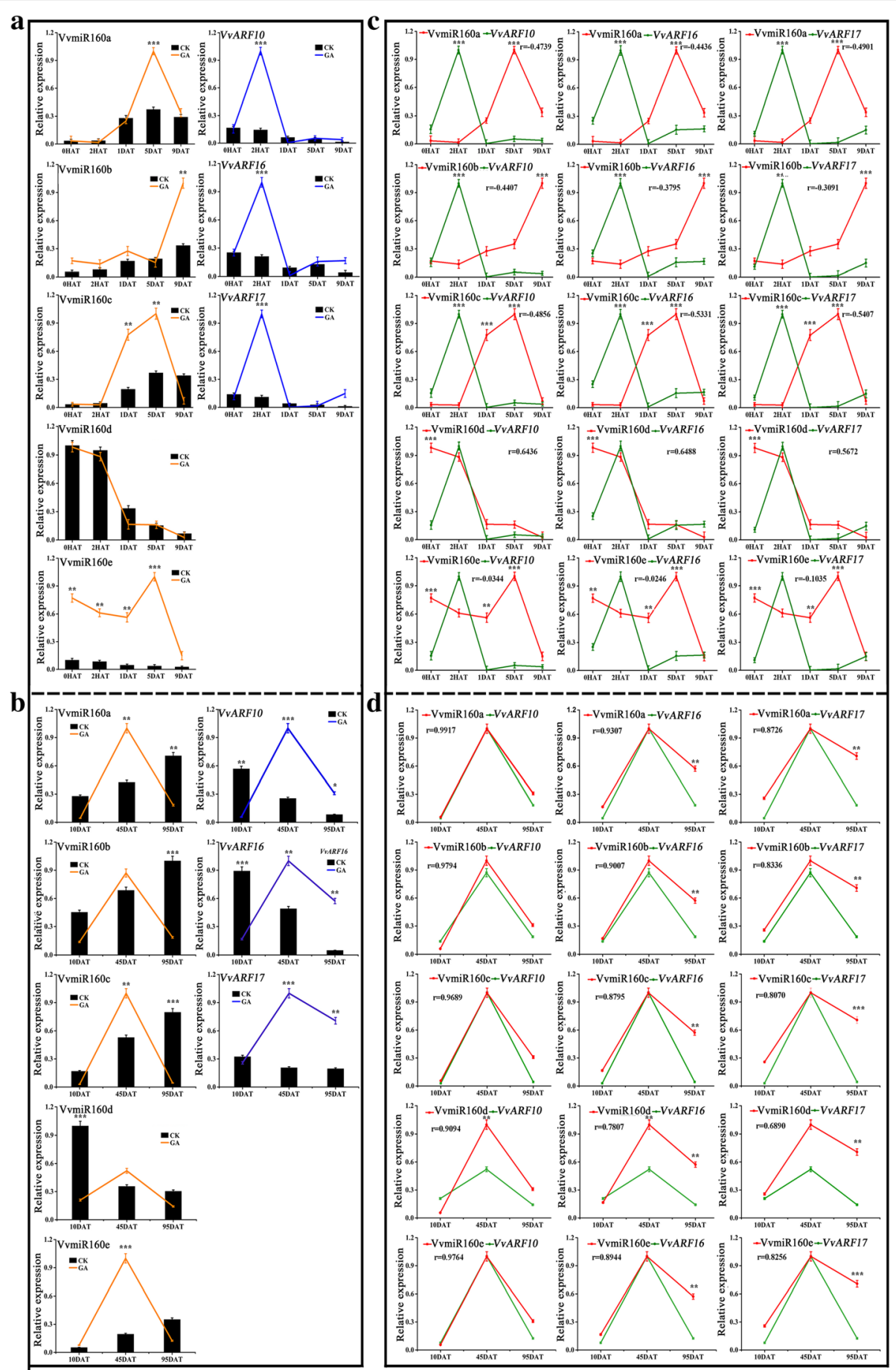

Fig. 9 Differential expression of VVmiR160 and VVARF target genes in response to GA application at different stages of floral and berry development. a Differential expression of VVmiR160s and their VVARF target genes in response to gibberellin (GA) treatment at different time points [0 $\mathrm{h}$ after treatment (OHAT), 2HAT, 1 day after treatment (1DAT), 5DAT, 9DAT] of floral development. b Differential expression of VVmiR160s and their VVARF target genes in response to gibberellin (GA) treatment at different time points (10DAT, 45DAT, 95DAT) of berry development. c Differential expression and Pearson correlation analysis of each member of VvmiR160 family and its respective VVARF target genes in response to GA treatment during floral development. d Differential expression and Pearson correlation analysis of each member of VVmiR160 family and its respective VVARF target genes in response to GA treatment during berry development. Each experiment was repeated three times. ANOVA test was used to identify significant differences, Asterisks indicate significant difference between GA-treated plants and respective untreated control (CK) plants at each time point as determined by Student's $t$-test $(* P<0.05$; $\left.{ }^{* *} P<0.01 ;{ }^{* *} P<0.001\right)$ 


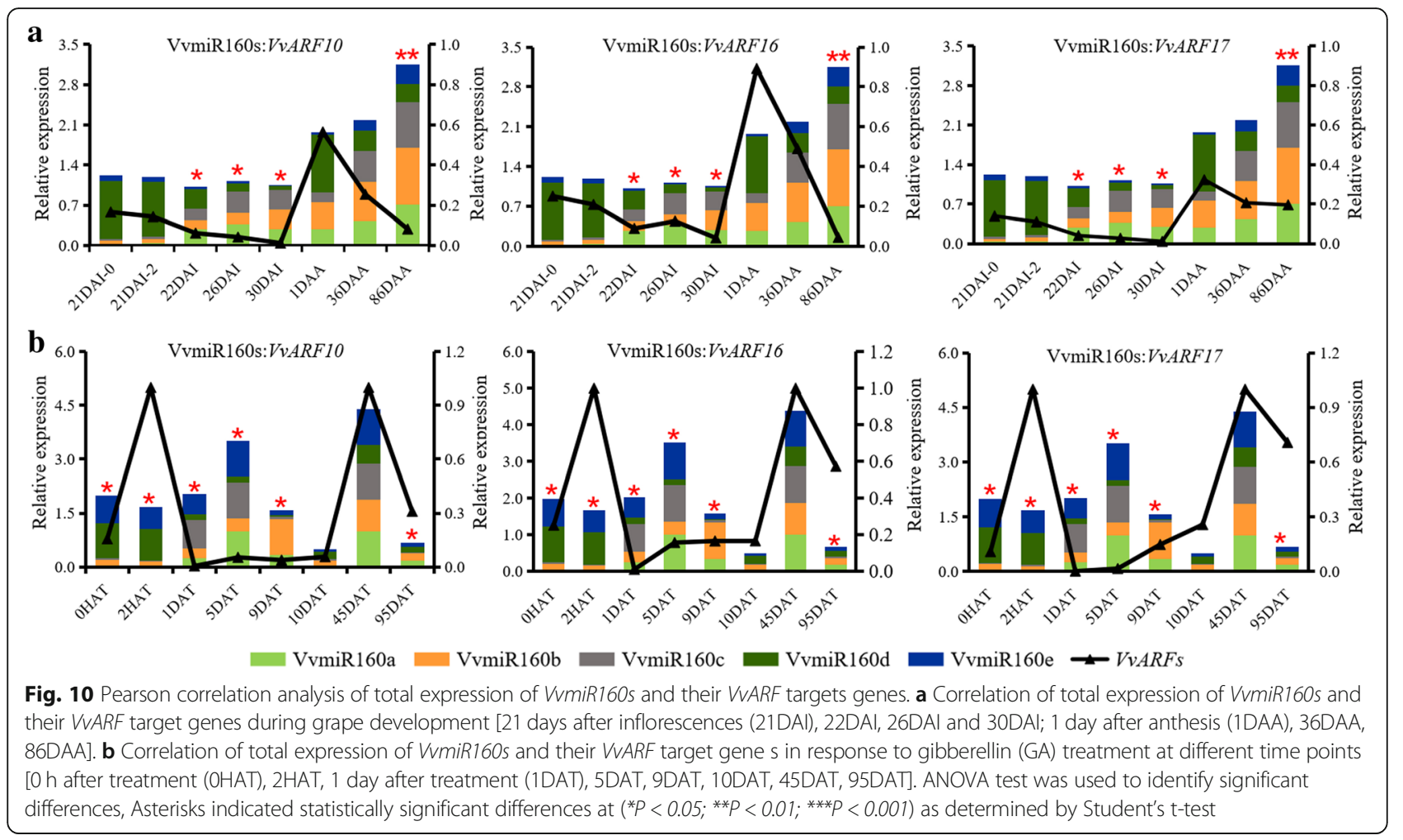

VvARF10/16/17 target genes mainly at the late stages (22DAI, 26DAI, and $30 \mathrm{DAI}$, marked with an asterisk) of grape floral development under control (Fig. 10a), and the total expression of VvmiR160s had negative correlations with $V v A R F 10 / 16 / 17$ target genes only at maturation (86DAA, marked with an asterisk) of grape berry development under control (Fig. 10a). While, under GA treatment, GA enhanced the negative regulatory effect of VvmiR160s on their target genes nearly at all stages (0HAT to 9DAT, marked with an asterisk) of grape floral development (Fig. 10b); however, no obvious effect of GA on the interaction of VvmiR160s and their target genes was observed during grape berry development (Fig. 10b). These results suggested that VvmiR160 family might possess some redundancy of functions, and indicated that GA is an essential factor for inducing the regulatory roles of VvmiR160s on their targets at short-term as well as long-term.

\section{Dynamic accumulation of cleavage products of ARF10/16/ 17 during GA-induced grape floral development}

Detecting the accumulation of cleavage products of VvmiR160s and VvARFs during grape floral development could be helpful to determine the spatio-temporal variation of their cleavage roles. Our modified RLM-RACE and developed PPM-RACE protocols together with qRT-PCR were employed to monitor their 3'- and 5 '-cleavage products under GA treatment. Results showed that GA treatment promoted greater accumulation of 3 'and $5^{\prime}$-cleavage products of $V v A R F 10 / 16 / 17$ in comparison with untreated control plants (Fig. 11). GA induced the highest accumulation of 3 ' - and 5 '-cleavage products of VvARF10/16/17 at 5DAT. However, GA slightly induced 3 ' - and 5 '-cleavage products at $2 \mathrm{HAT}$, indicating that GA exhibited a profound long-term effect on VvmiR160s' cleavage roles rather than a short-term one (Fig. 11). Although, the accumulation of 5 '-cleavage products of VvARF10/16/17 was almost similar to 3'-cleavage products at various phases in GA-treated and untreated control plants (Fig. 11), the 3 '-cleavage products were slightly higher, which might be due to the high stability of 3 '-cleavage products compared with 5 '-cleavage products as previously reported [30,33,34].

\section{Discussion}

The effects of exogenous GA application on the induction of flower development, seedless cultivars, berry enlargement and fruit ripening of grapevine have recently been reported $[18,20]$. However, the molecular mechanisms by which GA-signaling mediates flower and fruit set initiation in grapevines are still not fully understood. In recent years, miRNAs have been identified as a critical regulatory factor of gene expression through indirect, or direct transcriptional and post-transcriptional gene silencing to modulate the activity of the gene network underlying various developmental and stress-responsive programs $[3,9,14,21]$. 

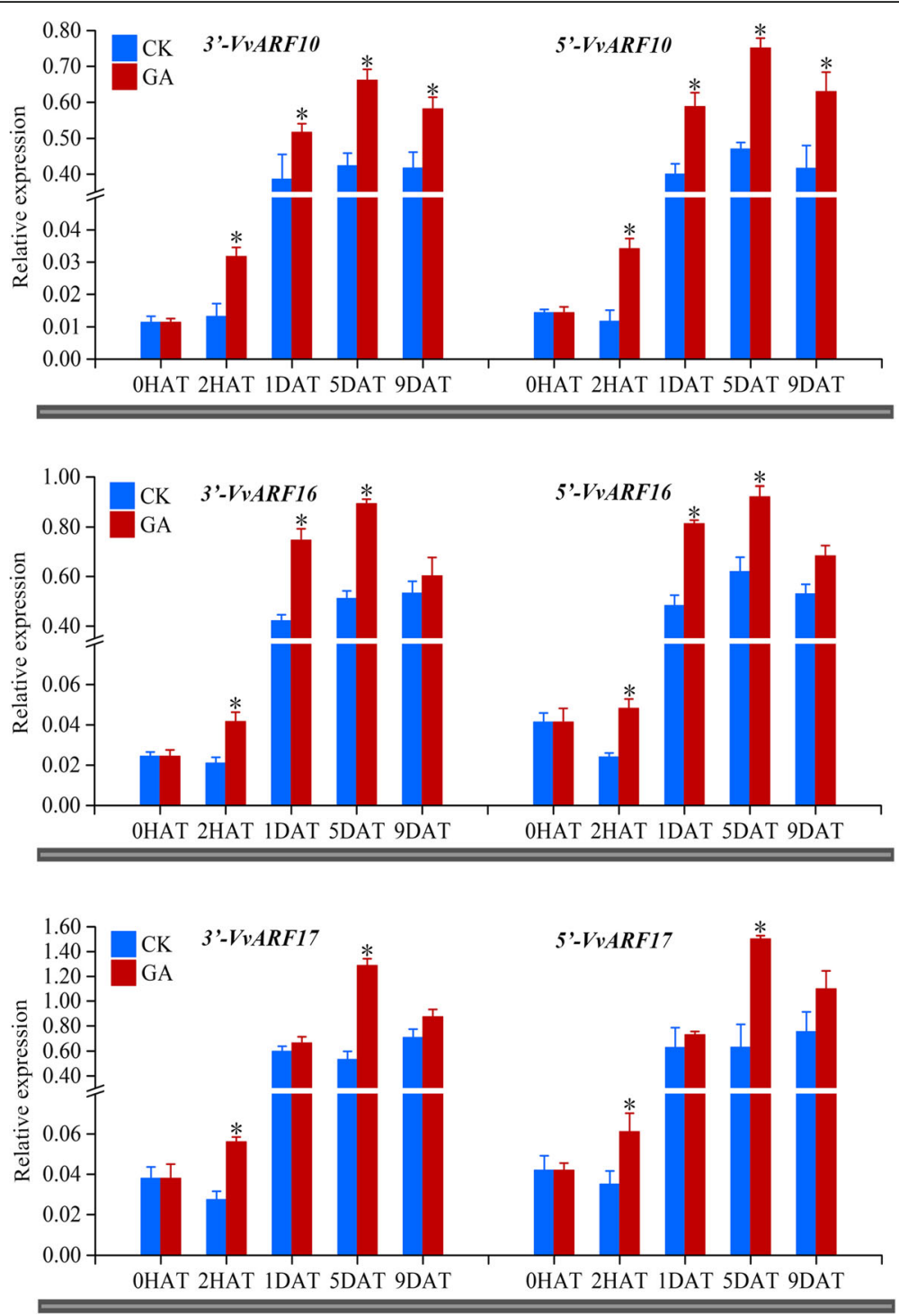

Fig. 11 Accumulation patterns of 3'- and 5'-end cleavage products of miR160s cleaved VVARF10/16/17 target genes in gibberellin (GA)-treated and untreated control (CK) plants at different stages of grape floral development. VVARFs-5'represents the accumulation of $5^{\prime}$-end cleavage products of VVARFs mediated by VVmiR160s using real-time QPCR; VVARFs-3' represents 3'-end cleavage products. Each experiment was repeated three times. [0 $\mathrm{h}$ after treatment (OHAT), 2HAT, 1 day after treatment (1DAT), 5DAT, 9DAT]. ANOVA test was used to identify significant differences, Asterisks indicated statistically significant differences at $\left({ }^{*} P<0.05 ;{ }^{* *} P<0.01\right)$ as determined by Student's t-test

Several miRNA families involved in the modulation of barley (Hordeum vulgare), Arabidopsis, cotton (Gossypium hirsutum) and tomato flower development have been identified and released in miRBase [10, 11, 24, 35-37]. For example, a recent study has reported that targeting of ARF10/16/17 by miR160 is indispensable for various aspects of AUX-mediated floral organ abscission as well as ovary patterning in tomato plant [24]. Similarly, a loss-of-function of Arabidopsis atmir160a plants changed the expression of $A R F 16 / 17$ and displayed different phenotypes, including irregular flowers, reduced fertility, aberrant seeds and floral organs inside siliques [17]. Our previous studies $[15,29]$ revealed that the exogenous application of $\mathrm{GA}_{3}$ induced the expression of several miRNA families, including miR160s and miR159s during grape floral development, suggesting that these miRNAs might play important roles in grape floral development and parthenocarpic process. In the present study, we utilized the grapevine parthenocarpy system to investigate the regulatory functions of VvmiR160s-VvARFs in response to GA-mediated grapevine floral development. The exogenous application of $\mathrm{GA}_{3}$ induced a variety of intriguing floral and berry morphology in 'Rosario Bianco' cultivar, including dark-yellow anthers, long filaments, short styluses, big ovaries and high seedless ratio, long berry spikes and grains longitudinal diameter relative to 
untreated control plants (Fig. 1a and b). Our result was in line with the recent study by Wang et al. [15] which demonstrated that $\mathrm{GA}_{3}$ application induced distinct floral morphology in grapevine cv. 'Zuijinxiang,' confirming that GA-signaling is a crucial factor for the positive regulation of grape parthenocarpy and fruit set development.

Based on our previous high-throughput sequencing of GA-induced grapevine parthenocarpy [15, 29], the precise sequences of $V v m i R 160 a / b / c / d / e$ and their VVARF10/16/17 target genes were isolated, sequenced and mapped into the corresponding chromosomes (Figs. 2 and 3). Although miRNAs are evolutionarily conserved among different plant species [38], our VvmiR160a/b exhibited two nucleotide shortage in comparison with homologous sequences from wine grapevine cv. 'Pinot Noir' in miRBase 21.0, while those of $V v m i R 160 \mathrm{c} / \mathrm{d} / \mathrm{e}$ were consistent in both grapevine cultivars (Fig. 2b). Our previous study revealed that about $85.2 \%$ of the known miRNAs had single cleavage sites on their target genes, whereas the remaining $14.8 \%$ of the known miRNAs had two to three cleavage sites, and these cleavage sites were mainly mapped on the 9th, 10th, or 11th nucleotide position from the 5 '-ends of the corresponding miRNAs [39]. According to the base-pair complementary degree between $V v m i R 160 s$ and $V v A R F s$ using 5'-RLM-RACE and 3'-PPM-RACE technology [30], we demonstrated that all $V v m i R 160 a / b / c / d / e$ had the same cleavage site on 9th position from the $5^{\prime}$-end (Fig. 7), whereas their corresponding cleave sites on VvARF10/16/17 were variables and located at the 1337th with 14/14, 1304th with $16 / 16$ and 1364th with $22 / 22$ from the $5^{\prime}$-end for VvARF10, VvARF16 and VvARF17, respectively (Fig. 7). These results suggested that the cleavage sites possessed the specific trait of miRNA sites' recognition, similar to previous reports that miRNAs cleaved their targets at specific sites from their $5^{\prime}$-ends like 9th, 10th and 11th [30, 33, 34]. PPM-RACE results further showed higher accumulation levels of 3 '-end cleavage products than those of RLM-RACE $5^{\prime}$-end cleavage products. The accumulation of 3 '-end cleavage products could be attributed to the rapid degradation of $5^{\prime}$-RNA fragment generated after miRNA-directed cleavage, while the $3^{\prime}$-RNA fragments are more stable, similar to previous reports in apple, orange, and peach [30, 38].

\section{Conserved regulatory roles of miR160 on their ARF target genes across diverse plant species}

The phylogenetic tree of our VvARFs and orthologues ones demonstrated an orthologous relationship between VvARFs and AtARFs, SlARFs and JrARFs (Fig. $4 a)$, suggesting that these ARF proteins might have descended from a common ancestor and can have conserved functions. The amino acid sequences of the three VvARF10/16/17 contained a conserved N-ter minal B3 and AUX_resp DBD, and C-terminal AUX/ IAA domain (CTD: domain III/IV), whereas VvARF17 and other orthologues ARF17 members showed the absence of C-terminal Aux/IAA domains (Fig. 4c). B3-type and AUX_resp DBD are essential for the interaction between ARFs and TGTCTC AuxREs, whereas AUX/IAA domain facilitates protein-protein interactions via homo- and hetero-dimerization among the members of both the ARF and Aux/IAA families [4042]. The Auxin_resp domain is an evolutionary step in plants, and freshwater algae ancestors of land plants are reported to contain precursor ARF-like proteins with III/IV and B3 domains but lack the Auxin_resp domain [22]. In addition, gene structure analysis indicated that the coding sequences of all the $V v A R F$ genes are disrupted by introns; however, the total number of introns was varied among different $V v A R F$ members (Fig. 4b). The exon-intron structures of most homologous $A R F$ genes had a similar distribution of exon-intron structures, which provide vital evidence to support the reliability of the phylogenetic analysis of VvARFs and their homologous ARFs in Arabidopsis, tomato and other species (Fig. 4a and b) and suggest a potential conserved function.

Furthermore, promoter motifs related to light, hormone, tissue-specific, circadian and stress-responsive cis-elements of VvmiR160s and their VVARF targeted genes exhibited high similarity, suggesting that their function is similar to a certain extent (Fig. 5a). Likewise, the promoter analysis of miR160s isolated from Dimocarpus longan showed that cis-elements responsive to stimuli such as light, hormone and circadian were also detected $[32,43]$. In the present work, all the promoter regions of VvmiR160a/c/d/e (except for VvmiR160b) and VvARFs contained cis-elements related to various hormonal response (GA, IAA, SA, ABA, MeJA and ET), indicating that VvmiR160s are associated with hormone transduction during grape development. Among these hormone-responsive motifs, GA-responsive cis-element was highly abundant in VvmiR160s and VvARFs, in addition, the transient expression experiments and GUS activity assays of VvmiR160c and VvARF10 promoters showed they were probably positively regulated by GA, which added further evidence regarding the essential roles of VvmiR160-VvARF pairs in grape development through GA-signaling. Likewise, number of copies of GA-responsive elements were also detected in the promoter regions of Glycine $\max (G m m i R 160 f / g / h)$ and Manihot esculenta (MemiR160a) and their GmARF6/8/ 17/18 and MeARF10 targeted genes, respectively [29, 44]. In general, these results clearly demonstrated the significant role of VvmiR160s in the regulation of GA-signaling through $V v A R F s$. 
Characterization of the expression patterns responsive to GA of various VvmiR160s:VvARF pairs during grape development

Multiple members of miRNA family and their target genes can form miRNA-mediated regulatory networks with some redundancy and complementarity functions, and different miRNA family members had diverse spatio-temporal expression modes [45]. Changes in the expression profiles of VvmiR160:VvARF pairs during grape development were presented in Fig. 8. Recent studies have shown that miR160s negatively regulate ARF10/16/17 in Arabidopsis, tomato, longan and populous [46-49]. However, the regulatory roles of miR160s in grape floral and berry development are unknown. In the present study, the spatio-temporal expression of different members of $V v m i R 160$ s showed that $V v m i R 160 a / b / c$ exhibited a significant increase in their expression levels during GA-mediated floral development (Fig. 9a). However, VvARF10/16/17 targeted genes displayed a reverse expression trend compared with $m i R 160 a / b / c$. Our result was in accordance with Lin et al. [32] demonstrated that GA treatment induced longan Dlo-miR160a/d expression and down-regulated DlARF10/16/17 during the middle and late stage of somatic embryogenesis. Similarly, foc mutant Arabidopsis seedling with a Ds transposon insertion in the 3 ' regulatory region of atmir160a exhibited down-regulation in atmir160a expression and up-regulation in AtARF10/16/17 in response to IAA treatment, and foc Arabidopsis mutant showed irregular flower phenotyping and reduced fertility [17]. Likewise, the knockdown of tomato SlmiR160 expression using a short tandem target mimic (STTM160) displayed abnormal floral organ abscission, which was associated with the up-regulation of SIARF10A/B and SlARF17 [24]. Our results and the above reports, clearly demonstrated that VvmiR160s are crucial for GA-induced grapevine floral development through the negative regulation of $V v A R F 10 / 16 / 17$ expression. In grape berries, the expression profiles of $\mathrm{VvmiR} 160 \mathrm{a} / \mathrm{b} / \mathrm{c}$ exhibited one upward trend along with berry development, and reached the high peak till maturation (Fig. 8b), likewise, the expression patterns of miR160a was gradually increased as fruit develops and ripens in blueberry [50]. In addition, our spatio-temporal expression of GA-induced grapevine shown that VvmiR160d expression was up-regulated at the early stage but down-regulated at the middle and later stage of GA-induced grape floral development, suggesting that VvmiR160d is acting as a repressor for grape floral development (Fig. 9a). Also, VvmiR160e expression displayed low expression level at all stages, implying that VvmiR160e has a minor role in grapevine floral and berry transition (Fig. 9a and b). We further carried out a comparative expression profiles of $V v m i R 160 s$ and $V v A R F$ targeted genes between GA-treated and untreated control (CK) grapevine to validate the significant role of GA treatment on grapevine floral/berry development through the VvmiR160-VvARF regulatory network (Fig. 9a and b). The expression trend of VvmiR160s and $V v A R F s$ at different stages under GA-treated and untreated grapevine plants was quite similar during grape floral development, however, GA treatment induced the expression of these respective genes much higher than their expression level in the untreated control plants (Fig. 9a). In contrast, GA treatment almost significantly up-regulated the expression of VvmiR160s and three VvARFs genes at berry expansion (Fig. 9b). It worth noting that IAA stimulates GA biosynthesis in pea pericarp $[51,52]$ through a process mediated by one or more signaling elements, and that GA metabolism interacts with IAA signaling independent of feedback regulation [53], when the AUX signaling pathway was blocked. These results could provide significant information for further gaining the functions of VvmiR160s and their target VvARFs during GA-induced grape parthenocarpy process.

\section{Conclusion}

This study examined the morphological changes of the 'Rosario Bianco' floral and berry development during grapevine parthenocarpy process-induced by the exogenous $\mathrm{GA}_{3}$ application. The precise sequences of VvmiR160s in 'Rosario Bianco' grape flowers and their VvARF10/16/17 target genes were predicted, cloned and verified. Furthermore, the conserved and diversification phylogenetic relationships of VvARF with other orthologues from various plant species were carried out. All VvmiR160s (except VvmiR160b) and VvARF10/16/17 had showed the common cis-elements responsive to $\mathrm{GA}$, and this was confirmed during our experiments, which support their function in GA-mediated grape parthenocarpy. The spatio-temporal expression and correlation analysis indicated that $\mathrm{VvmiR} 160 \mathrm{a} / \mathrm{b} / \mathrm{c}$ are the key factors that negatively regulate $V v A R F 10 / 16 / 17$ target genes during GA-induced grapevine floral and berry development. The negative regulation of $V v A R F 10 / 16 / 17$ expression by $V v m i R 160 a / b / c$ as key regulatory factors was critical for GA-mediated grape parthenocarpy, and provided a significant step forward in understanding the molecular mechanisms of VvmiR160s and their VvARFs for molecular breeding of high-quality seedless berry. Further studies are required to identify the specific downstream genes that are targeted by the corresponding ARFs in each process.

\section{Methods}

Plant materials and $\mathrm{GA}_{3}$ treatment

Basing on the preliminary study and the necessity of performing the related research work, 6-year-old trees of grapevine cv 'Rosario Bianco' (Vitis vinifera), an elite grape cultivar widely cultivated in China, were used in 
this study. The plant materials were grown under common field conditions at the Jiangsu Vocational College of Agriculture and Forestry grape farm, Jurong, China (our partners, we have a long-term relationship). Referencing to the production experience and variety characteristics, the grape clusters were soaked in $50 \mathrm{mg} \mathrm{L}^{-1} \mathrm{GA}_{3}$ (dissolved in $0.2 \%$ Tween-20) for $30 \mathrm{~s}$, and water-treated grape clusterswere used as a control at 21 days after inflorescences. The GA-treated plants reached anthesis at 9 days after treatments. Inflorescence and berry samples were randomly collected from different branches of different treatment groups at different time points $[0 \mathrm{~h}, 2 \mathrm{~h}$, $1,5,9,10,45$ and 95 days after treatment (OHAT, 2HAT, 1DAT, 5DAT, 9DAT, 10DAT, 45DAT and 95DAT respectively). Samples were used for phenotypic characters, and the other part of samples were immediately frozen in liquid nitrogen and stored at $-80^{\circ} \mathrm{C}$ until use.

RNA extraction, high-molecular-weight RNA, lowmolecular-weight RNA isolation and CDNA synthesis

Total RNA was isolated from $200 \mathrm{mg}$ grapevine tissues mentioned above using our modified CTAB method [30]. Low-molecular-weight RNA (LMW RNA) and high-molecular-weight RNA (HMW RNA) were separated with $4 \mathrm{M} \mathrm{LiCl}$. The mixtures of diverse stage LMW RNA samples were loaded into Poly(A) tails using Poly(A) Tailing kit from TAKARA. The Poly(A)-tailed LMW RNAs were further ligated to $5^{\prime}$ adapter (5'-CGACUGGAGCACGAGGACACUGACAUGGA CUGAAGGAGUAGAAA-3') using T4 RNA ligase (Invitrogen, Carlsbad, CA). Then, these LMW RNAs with Poly (A) and 5'-adapter were reversetranscripted into cDNAs for miR-RACE clone. Mixtures of diverse stage HMW RNA samples were directly reverse transcripted into cDNAs for qRT-PCR. All cDNAs were stored at $-80{ }^{\circ} \mathrm{C}$ before use.

The clone of the precise sequences of VvmiR160s in the floral tissues of grape cv. 'Rosario Bianco' by miR-RACE The mixtures of diverse stage LMW RNA samples were loaded into Poly(A) tails using Poly(A) Tailing kit from TAKARA. The Poly(A)-tailed LMW RNAs were further ligated to $5^{\prime}$ adapter (5'-CGACUGGAGCACGAGGACA CUGACAUGGACUGAAGGAGUAGAAA-3') using T4 RNA ligase (Invitrogen, Carlsbad, CA). Then, these LMW RNAs with Poly (A) and 5'-adapter were reverse transcripted into cDNAs for clones of VvmiR160 sequences by miR-RACE [30].

Prediction of the targets for VvmiR160s, identification of the degree of complementarity and related parameters of VvmiR160s:VvARF pairs

Based on the precise sequences of five VvmiR160 members, we employed Multiple Align (BioXM software) to align these sequences, revealing two unique mature sequences (VvmiR160a/b and VvmiR160c/d/e). The miRNA unique sequences were further used as the benchmark to blast search in grape transcript database V2 (http://genomes.cribi.unipd.it/grape/) for prediction of target genes for VvmiR160s. Furthermore, the identification of the degree of complementarity, action modes, and other related parameters were characterized using the psRNATarget software (http://plantgrn.noble.org/ psRNATarget/).

\section{Mapping of mRNA cleavage sites using RLM-RACE and PPM-RACE}

To map miRNA-mediated cleavage products, our earlier modified RLM-RACE and developed PPM-RACE were employed to determine their $33^{\prime}$ - and 5'-end cleavage products. Based on our previous report [30], HMW RNAs have been added to the Poly(A) (Ambion, Austin, TX) and ligated to $5^{\prime}$ adapter (5'-CGACUGGAGCACGAGGACA CUGACAUGGACUGAAGGAGUAGAAA-3') using T4 RNA ligase (Invitrogen, Carlsbad, CA), respectively. Then Poly (A)-tailed HMW RNA and adapter-ligated HMW RNA were recovered by phenol/chloroform extraction followed by ethanol precipitation. Poly (A)-tailed HMW RNA and adapter-ligated HMW RNA were further reverse transcripted into cDNAs for PPM-RACE and RLMRACE. The amplification products were gel purified, cloned, and sequenced, and at least eight independent clones were sequenced.

\section{Distribution of VvmiR160s and target VvARF genes in the precursors/chromosomes}

The precursors of VvmiR160s were downloaded from the plant miRBase 21.0 (http://www.mirbase.org/). Mfold online tool (http://unafold.rna.albany.edu/) were applied to fold their hairpin structures. All five VvmiR160s and three $V V A R F$ genes were mapped to grapevine chromosomes based on information available at the Grape Genome CRIBI website (http://genomes.cribi.unipd.it/grape/). The map was drafted using MapInspect software (http://www. plantbreeding.wur.nl/uk/software- mapinspect.html).

\section{Phylogenetic analysis}

The software MEGA version 7.0.21 and ClustalX2 were employed to conduct the phylogenetic analysis. Homologs of VvARF10, VvARF16, VvARF17 were identified by a blast search in the NCBI databases (https://blast.ncbi. nlm.nih.gov/Blast.cgi) using amino acid sequences of VvARF10/16/17. Multiple alignmentswere performed using ClustalX2, and the unrooted phylogenetic trees were constructed with MEGA 7.0.21 software using the Neighbor-Joining method, and the bootstrap test was carried out with 1000 replications. 


\section{Analysis and distribution of exon/intron structures of ARFs in nine species}

The exon/intron organization of $V v A R F$ genes was determined by comparing predicted coding sequences with their corresponding genomic sequences using the GSDS software (http://gsds.cbi.pku.edu.cn).

\section{Protein domains and their physicochemical properties analysis of target $A R F s$ in various species}

The Domains \& Structures website (https://www.ncbi. nlm.nih.gov/guide/domains- structures/) were used to analyze protein domains of target genes, and their homologs sequences. Then, the IBS (Illustrator for Biological Sequences) software version 1.0.3 was used to draw the orthodox domains of ARF proteins. Besides, all target ARF protein sequences were considered and used in further analysis. Number of amino acids, isoelectric point, aliphatic index, grand average of hydropathicity, the molecular weight of deduced polypeptides were calculated by using tools provided at the ExPasy website (http://web.expasy.org/protparam/).

\section{Motif composition analysis of ARF proteins in nine species}

The identification of motifs in the ARF protein sequences was performed with the MEME 5.05 online program (http://meme-suite.org/tools/meme). The optimized parameters of MEME were employed as follows: number of repetitions, any; maximum number of motifs, 20; and the optimum width of each motif, between 6 and 50 residues.

\section{Cis-element analysis of the promoters from VvMIR160s and target VvARFs}

From the grape genoscope database (http://www.genoscope.cns.fr/externe/GenomeBrowser/Vitis/), we obtained the promoters (approximately $1500 \mathrm{bp}$ upstream of genes) of VvMIR160 (VvmiR160's precursors) and VvARFs, and employ the Plantcare software (http://bioinformatics.psb. ugent.be/webtools/plantcare/html/) to predict the motif elements in these promoters.

\section{Construction of the expression vector and Agrobacterium- mediated tobacco transient transformation}

The promoter sequences of VvMIR160c and VvARF10 were isolated. The primers used were described in Additional file 6: Table S4. To develop pBI121-p1VvMIR160c-GUS and pBI121-p1VvARF10-GUS constructs, $1.5 \mathrm{~kb}$ promoter regions of $V v M I R 160 \mathrm{c}$ and $V v A R F 10$ were independently cloned and fused with GUS reporter gene to replace the $35 S C a M V$ promoter in pBI121 binary vector. With respect to pBI121-p2VvMIR160c-GUS construct, $1442 \mathrm{bp}$ promoter 2 fragment region of $V v M I R 160 c$ without including the GA response element (59-1500 bp) was cloned into pBI121 to replace the
$35 S C a M V$ promoter and was used to drive the GUS reporter gene. Similarly, for pBI121-p2VvARF10-GUS construct, 937 bp promoter 2 fragment region of VvmiR160c without GA response element (564-1500 bp) was cloned into pBI121 to replace the $35 \mathrm{SCaMV}$ promoter and was used to drive the GUS reporter gene.

A single colony of Agrobacterium tumefaciens EHA105 (containing the recombinant plasmid) was cultured in YEB liquid medium supplied with rifampin $\left(50 \mu \mathrm{g} \mathrm{mL}^{-1}\right)$ and kanamycin $\left(50 \mu \mathrm{g} \mathrm{mL}^{-1}\right)$. After culturing, the bacteria were pelleted and resuspended in suspension buffer (10 $\mathrm{mM}$ MES, $10 \mathrm{mM} \mathrm{MgCl} 2$, $\mathrm{pH}$ 5.6). The bacterial.

suspension was then adjusted to OD600 $=0.5$, and $100 \mu \mathrm{mol} \mathrm{L}^{-1}$ acetosyringone was added before the suspension was used for infiltration, and left to stand at room temperature for $4 \mathrm{~h}$. The leaves of 6-week-old tobacco plants were infiltrated with the bacterial suspension by injection. Tiny holes were made in the tobacco leaves using syringe needles. The bacterial suspension was then injected into those tiny holes with a needleless syringe. The infiltrated seedlings were then moved back to the environmental chamber and kept in the dark for 3 d. Treatment on tobacco leaves with $30 \mu \mathrm{M}$ GA and $50 \mu \mathrm{M}$ GA were performed 2 days later. The uniformly sized leaves were used in infiltration experiment, and the experiment was repeated three times.

\section{GUS assay}

The infiltrated leaves were punched and then histochemical GUS staining of infiltrated leaves was performed as described by Jia et al. [54]. Leaves were then immersed in ethanol to remove Chl. Quantification of GUS activity was determined using the fluorometric 4-methylumbelliferyl-b-D-glucuronide (MUG) method. One unit of GUS activity was defined as $1 \mathrm{nM}$ of 4-methylumbelliferon (4-MU) generated per minute per milligram of soluble protein. Three leaves were infiltrated for each construct in each independent experiment, and then combined to detect GUS activity.

\section{Expression analysis of VvmiR160s by qRT-PCR}

The template for quantitative real-time polymerase chain reaction (qRT-PCR) was the cDNA for poly(A)-tailed small RNA mentioned above. To amplify the VvmiR160a/b/ c/d/e from the reverse-transcribed cDNAs, we used the precise sequences of VvmiR160 family members as the forward primer and R16328 (ATTCTAGAGGCCGAGGCGGCCGACATG) as the reverse primer [30]. qRT-PCR was performed with SYBR Premix Ex Tap ${ }^{\mathrm{Tw}}$ kit (TaKaRa, Dalian, China), using Light Cycler 480 II (Roche, Switzerland). PCR cycling conditions consisted of an initial denaturation step at $95^{\circ} \mathrm{C}$ for 30s, followed by 40 cycles at $60^{\circ} \mathrm{C}$ for 20 s and $95^{\circ} \mathrm{C}$ for $5 \mathrm{~s}$. The $5.8 \mathrm{~s}$ rRNA was used as a reference gene. 
Relative expression level was calculated with the formula $2^{-\Delta \Delta C T}=$ normalized expression ratio. Each PCR assay was carried out by three biological replicates, and each replicate corresponded to three repeats of separate experiments. All primers were listed in Additional file 6: Table S4.

\section{Real-time PCR of the VvARFs expression}

The expression of $V v A R F 10 / 16 / 17$ was assayed by qRT-PCR as previously described [30]. The reverse transcription product was amplified using gene-specific primers that overlapped the known or predicted cleavage site. Reactions were performed in triplicates using the Light Cycler 480 II. The Actin gene was used as a reference gene in the qRT-PCR detection of mRNAs. Relative expression level was calculated with the formula $2^{-\Delta \Delta C T}$ = normalized expression ratio. Each PCR assay was carried out in three biological replicates. The experiments were set the three repeats. All primers were listed in Additional file 6: Table S4.

\section{Statistics}

ANOVA test was used to identify significant differences in floral morphology and gene expression between $\mathrm{GA}_{3}$-treated and untreated grape plants. Asterisks indicate a significant difference between GA-treated plants and respective untreated control (CK) plants at each time point as determined by Student's $t$-test $\left({ }^{*} P<0.05 ;{ }^{* *} P<0.01\right)$.

\section{Additional files}

Additional file 1: Figure S1. Phylogenetic tree of three ARF domains based on an alignment of grapevine and Arabidopsis. The phylogenetic tree was generated with MEGA 7.0.21 software using the neighborjoining method. Bootstrap values from 1000 replicates are indicated at each branch. (TIF 2006 kb)

Additional file 2: Figure S2. The open reading frame (ORF) and amino acid sequences of AUXIN RESPONSIVE FACTOR (VVARF)10/16/17 in grapevine. Asterisk indicates the stop codon. (TIF 6656 kb)

Additional file 3: Table S1. Protein domains of ARFs and their physicochemical properties. Noaa, Number of amino acids; pl, isoelectric point; Ai, aliphatic index; GRAVY, grand average of hydropathicity; MW, molecular weight. (DOCX $21 \mathrm{~kb}$ )

Additional file 4: Table S2. All the motif information of VVMIR160s precursor genes and their targeted VVARF genes' promoter.The Plantcare software (http://bioinformatics.psb.ugent.be/webtools/plantcare/html/) was used to predict the motif elements of these genes' promoter. (DOCX $24 \mathrm{~kb}$ )

Additional file 5: Table S3. Motif elements related to hormone signals of MIR160s precursors' genes and their targeted ARFs pairs in grapevine. The Plantcare software (http://bioinformatics.psb.ugent.be/webtools/ plantcare/html/) was used to predict the motif elements of these genes' promoter. (DOCX $16 \mathrm{~kb}$ )

Additional file 6: Table S4. List of the primers used for experiments. (DOCX $16 \mathrm{~kb})$

\section{Abbreviations}

ABA: Abscisic acid: ARE: AUXIN RESPONSIVE FACTOR: AUX: Auxin: AuxREs: AUXIN RESPONSEELEMENTs; Chr: Chromosome; ET: Ethylene; foc: floral organs in carpel; GA: Gibberellin; HMW RNA: High-molecular-weight RNA; IAA: Indole acetic acid; LMW RNA: Low-molecular-weight RNA; MeJA: Methyl jasmonate; ORF: Open reading frame; PPM-RACE: Poly (A) polymerase -mediated 3 ' rapid amplification of cDNA ends; RLM-RACE: RNAligase-mediated Rapid Amplification of cDNA Ends; SA: Salicylic Acid; SLR1: SLENDER RICE 1; STTM: Short tandem target mimic

\section{Acknowledgments}

The authors are grateful for the research laboratory facilities provided by the College of Horticulture, Nanjing Agricultural University, Nanjing, China. MA Professor would like to thank Tottori University, Japan, and Aswan University, Egypt, for this collaborative research work.

\section{Funding}

This work was financially supported by the Ministry of Science and Technology of China (Nos. 2018YFD1000106); Jiangsu Provincial Natural Science Fund (BK20180113 and BK20160587) and the Central University basic scientific research business fee independent innovation major project (NATURAL SCIENCE, KYTZ201602). The funding agencies provided only the experimental cost and publication fee for this work. However, the experimental design and data collection and analysis were managed by the contributing authors.

\section{Availability of data and materials}

All VvmiR160s and VVARF cDNA are available upon request. All primers and amino acid sequences used in this study are listed in Additional files 3, 4, 5 and 6: Tables S1-S4.

\section{Authors' contributions}

CW conceived and designed the study, WZ collected the samples, conducted the expression analysis of miRNAs and their targets, MA and SJ carried out the molecular mechanism analysis. JH and TZ performed the promoter element analysis of MIRNAs and their targets. LG participated in the activity verification of promoters in supplementary experiments and paper modification. CW validated their cleavage roles. WZ, MA, HJ and CS performed data analysis and figure preparation. WZ wrote the manuscript. CW, MA and JF revised the manuscript. All authors read and approved the final manuscript.

\section{Ethics approval and consent to participate}

The plants used in our study are not endangered species. Plant sample collection and transgenic plants were performed in accordance with the local legislation in China.

\section{Consent for publication}

Not applicable.

\section{Competing interests}

The authors declare that they have no competing interests.

\section{Publisher's Note}

Springer Nature remains neutral with regard to jurisdictional claims in published maps and institutional affiliations.

\section{Author details}

${ }^{1}$ College of Horticulture, Nanjing Agricultural University, Nanjing 210095 , China. ${ }^{2}$ Department of Botany, Faculty of Sciences, Aswan University, Aswan 81528, Egypt. ${ }^{3}$ Arid Land Research Center, Tottori University, Tottori 680-001, Japan. ${ }^{4}$ Department of Plant Science, School of Agriculture and Biology, Shanghai Jiao Tong University, Shanghai, China.

Received: 29 October 2018 Accepted: 14 March 2019

Published online: 21 March 2019

References

1. Camposrivero G, Osoriomontalvo P, Sánchezborges R, Uscamas R, Duarteaké F, Dela PC. Plant hormone signaling in flowering: an epigenetic point of view. J Plant Physiol. 2017;214:16-27.

2. Blázquez MA, Weigel D. Integration of floral inductive signals in Arabidopsis. Nature. 2000;404:889-92. 
3. Spanudakis $\mathrm{E}$, Jackson $\mathrm{S}$. The role of microRNAs in the control of flowering time. J Exp Bot. 2014;65:365-80.

4. Wang JW. Regulation of flowering time by the miR156-mediated age pathway. J Exp Bot. 2014;65:4723-30.

5. Kazan K, Lyons R. The link between flowering time and stress tolerance. J Exp Bot. 2015;67:47-60.

6. Song GQ, Chen QX. Comparative transcriptome analysis of nonchilled, chilled, and late-pink bud reveals flowering pathway genes involved in chilling-mediated flowering in blueberry. BMC Plant Biol. 2018;18:98.

7. Park M, Krause C, Karnahl M, Reichardt I, Kasmi EF, Mayer U, et al. Concerted action of evolutionarily ancient and novel SNARE complexes in floweringplant cytokinesis. Developmental cell. 2018;44:500-511.e4.

8. Yamaguchi A, Abe M. Regulation of reproductive development by noncoding RNA in Arabidopsis: to flower or not to flower. J Plant Res. 2012;125: 693-704.

9. Luo Y, Guo ZH, Li L. Evolutionary conservation of microRNA regulatory programs in plant flower development. Dev Biol. 2013;380:133-44.

10. Hong YQ, Jackson S. Floral induction and flower formation-the role and potential applications of miRNAs. Plant Biotechnol J. 2015;13:282-92.

11. Teotia S, Tang GL. To bloom or not to bloom: role of microRNAs in plant flowering. Mol Plant. 2015;8:359-77.

12. WangC HJ, Liu CH, Korir NK, Emrul K, Shangguan LF, et al. Identification of microRNAs from Amur grape (Vitis amurensis Rupr.) by deep sequencing and analysis of microRNA variations with bioinformatics. BMC Genomics. 2012:13-122.

13. Suzuki HI, Young RA, Sharp PA. Super-enhancer-mediated RNA processing revealed by integrative microRNA network analysis. Cell. 2017;168:1000-14.

14. Abdelrahman M, Jogaiah S, Burritt DJ, Tran LP. Legume genetic resources and transcriptome dynamics under abiotic stress conditions. Plant Cell \& Environment. 2018;In press.

15. Wang C, Jogaiah S, Zhang WY, Abdelrahman M, Fang J. G. Spatio-tempora expression of miRNA159 family members and their GAMYB target gene during the modulation of gibberellin-induced grapevine parthenocarpy. J Exper Botany. 2018;In press.

16. Aukerman MJ, Sakai H. Regulation of flowering time and floral organ identity by a MicroRNA and its APETALA2-like target genes. Plant Cell. 2003; 15:2730-41.

17. Liu XD, Huang J, Wang Y, Khanna K, Xie ZX, Owen HA, et al. The role of floral organs in carpels, an Arabidopsis loss-of-function mutation in MicroRNA160a, in organogenesis and the mechanism regulating its expression. Plant J. 2010;62:416-28.

18. Cheng CX, Jiao C, Singer SD, Gao M, Xu XZ, Zhou YM, et al. Gibberellininduced changes in the transcriptome of grapevine (Vitis labrusca $\times V$. vinifera) cv. Kyoho flowers. BMC Genomics. 2015:16-128.

19. Liu X, Xu T, Dong XF, Liu YD, Liu ZH, Shi ZH, et al. The role of gibberellins and auxin on the tomato cell layers in pericarp via the expression of ARFs regulated by miRNAs in fruit set Acta. Physiologiae Plantarum. 2016;38:1-11.

20. Wang MQ, Sun X, Wang C, Cui LW, Chen LD, Zhang CB, et al. Characterization of miR061 and its target genes in grapevine responding to exogenous gibberellic acid. Functional \& Integrative Genomics. 2017:17:537-49.

21. Achard P, Herr A, Baulcombe DC, Harberd NP. Modulation of floral development by a gibberellin-regulated microRNA. Development. 2004;131: 3357-65.

22. Guilfoyle TJ, Hagen G. Auxin response factors. Curr Opin Plant Biol. 2007;10: 453-60.

23. Okushima Y, Fukaki H, Onoda M, Theologis A, Tasakaa M. ARF7 and ARF19 regulate lateral root formation via direct activation of $\angle B D / A S L$ genes in Arabidopsis. Plant Cell. 2007;19:118-30.

24. Damodharan S, Zhao D, Arazi T. A common miRNA160-based mechanism regulates ovary patterning, floral organ abscission and lamina outgrowth in tomato. Plant J Cell Mol Biol. 2016;86:458.

25. Goetz M, Hooper LC, Johnson SD, Rodrigues JC, Vivian-Smith A, Koltunow AM. Expression of aberrant forms of AUXIN RESPONSE FACTOR8 stimulates parthenocarpy in Arabidopsis and tomato. Plant Physiol. 2007;145:351-66.

26. De JM, Mariani $\mathrm{C}$, Vriezen $\mathrm{WH}$. The role of auxin and gibberellin in tomato fruit set. J Exp Bot. 2009;60:1523-32.

27. Jung CJ, Hur YY, Yu HJ, Noh JH, Park KS, Lee HJ. Gibberellin application at pre-bloom in grapevines down-regulates the expressions of VVIAA9 and V VARF7, negative regulators of fruit set initiation, during parthenocarpic fruit development. PLoS One. 2014;9:e95634.
28. Rubiosomoza I, Weigel D. Coordination of flower maturation by a regulatory circuit of three MicroRNAs. PLoS Genet. 2013;9:e1003374.

29. Han J, Fang JG, Wang C, Yin YL, Sun X, Leng XP, et al. Grapevine microRNAs responsive to exogenous gibberellin. BMC Genomics. 2014;15:111.

30. Wang C, Han J, Korir NK, Wang XC, Liu H, Li XY, et al. Characterization of target mRNAs for grapevine microRNAs with an integrated strategy of modified RLM-RACE, newly developed PPM-RACE and QPCRs. J Plant Physiol. 2013;170:943-57.

31. Wang M, Vannozzi A, Wang G, Liang YH, Battista GT, Sara Z, et al. Genome and transcriptome analysis of the grapevine (Vitis vinifera L.) WRKY gene family. Horticulture Res. 2014;1:14016.

32. Lin $Y L$, Lai $Z X$, Tian $Q$, Lin $L X$, Lai RL, Yang MM, et al. Endogenous target mimics down-regulate miR160 mediation of ARF10, -16 , and -17 cleavage during somatic embryogenesis in Dimocarpus longan Lour. Front Plant Sci. 2015;6:e219.

33. Sun X, Korir NK, Han J, Shangguan LF, Kayesh E, Leng XP, et al. Characterization of grapevine microR164 and its target genes. Mol Biol Rep. 2012;39:9463-72.

34. Zhang YP, Han J, Yu ML, Ma RJ, Pervaiz T, Fang JG. Characterization of target mRNAs for Prunus persica microRNAs using an integrated strategy of PLM-RACE, PPM-RACE and GRT-PCR. Scientia Horticulturae. 2014;170:8-16.

35. Nag A, King S, Jack T. miR319a targeting of TCP4 is critical for petal growth and development in Arabidopsis. Proc Natl Acad Sci U S A. 2009;106:22534-9.

36. Liu N, Tu LL, Wang LC, Hu HY, Xu J, Zhang XL. MicroRNA 157-targeted SPL genes regulate floral organ size and ovule production in cotton. BMC Plant Biol. 2017;17:7.

37. Tripathi RK, Bregitzer P, Singh J. Genome-wide analysis of the SPL/miR156 module and its interaction with the AP2/miR172 unit in barley. Sci Rep. 2018:8:7085.

38. Sun X, Zhang YP, Zhu XD, Korir NK, Tao R, Chen W, et al. Advances in identification and validation of plant microRNAs and their target genes. Physiol Plant. 2014;152:203-18.

39. Wang $C$, Leng $X P$, Zhang $Y Y$, Kayesh E, Zhang YP, Sun $X$, et al. Transcriptome-wide analysis of dynamic variations in regulation modes of grapevine microRNAs on their target genes during grapevine development. Plant Mol Biol. 2014:84:269-85.

40. Wan SB, Li WL, Zhu YY, Liu ZM, Huang WD, Zhang JC. Genome-wide identification, characterization and expression analysis of the auxin response factor gene family in Vitis vinifera. Plant Cell Rep. 2014;33:1365-75.

41. Lavy M, Prigge MJ, Tao S, Shain S, Kuo A, Kirchsteiger K, et al. Constitutive auxin response in Physcomitrella reveals complex interactions between aux/ IAA and ARF proteins. Elife. 2016;5:e13325.

42. Parcy F, Vernoux T, Dumas R. A glimpse beyond structures in auxindependent transcription. Trends Plant Sci. 2016;21:574-83.

43. Zhou XF, Ruan JH, Wang GD, Zhang WX. Characterization and identification of MicroRNA core promoters in four model species. PLoS Comput Biol. 2007;3:e37.

44. Pinweha N, Asvarak T, Viboonjun U, Narangajavana J. Involvement of miR160/miR393 and their targets in cassava responses to anthracnose disease. J Plant Physiol. 2015;174:26-35.

45. Wang BJ, Wang J, Wang C, Shen WB, Jia HF, Zhu XD, et al. Study on modes of expression and cleavage role of miR156b/c/d and its target gene $V_{V}-S P L 9$ during the whole growth stage of grapevine. J Hered. 2016;107:626-34.

46. Kumar R, Tyagi AK, Sharma AK. Genome-wide analysis of auxin response factor (ARF) gene family from tomato and analysis of their role in flower and fruit development. Mol Genet Genomics. 2011;285:245.

47. Yang $C X, X u M, X u a n ~ L$, Jiang $X M$, Huang MR. Identification and expression analysis of twenty ARF genes in Populus. Gene. 2014;544:134-44.

48. Zouine M, Fu YY, Chateignerboutin AL, Chateigner-Boutin AL, Mila I, et al. Characterization of the tomato ARF gene family uncovers a multi-levels post-transcriptional regulation including alternative splicing. PLoS One. 2014;9:e84203.

49. Wang B, Xue JS, Yu YH, Liu SQ, Zhang JX, Yao XZ, et al. Fine regulation of ARF17 for anther development and pollen formation. BMC Plant Biol. 2017;17:243.

50. Hou YM, Zhai LL, Li XY, Xue Y, Wang JJ, Yang PJ, et al. Comparative analysis of fruit ripening-related miRNAs and their targets in blueberry using small RNA and degradome sequencing. Int J Mol Sci. 2017;18:2767.

51. Huizen RV, Ozga JA, Reinecke DM, Twitchin B, Mander LN. Seed and 4chloroindole-3-acetic regulation of gibberellin metabolism in pea pericarp. Plant Physiol. 1995;109:1213-7. 
52. Ozga JA, Yu J, Reinecke DM. Pollination-, development-, and auxin-specific regulation of gibberellin 3 $\beta$-hydroxylase gene expression in pea fruit and seeds. Plant Physiol. 2003;131:1137-46.

53. Frigerio M, Alabadi D, Perez-Gomez J, García-Cárcel L, Phillips AL, Hedden P, et al. Transcriptional regulation of gibberellin metabolism genes by auxin signaling in Arabidopsis. Plant Physiol. 2006;142:553-63.

54. Jia HF, Jiu ST, Zhang C, Wang C, Pervaiz T, Liu ZJ, et al. Abscisic acid and sucrose regulate tomato and strawberry fruit ripening through the abscisic acid-stress-ripening transcription factor. Plant Biotechnol J. 2016;14:2045-65.

Ready to submit your research? Choose BMC and benefit from:

- fast, convenient online submission

- thorough peer review by experienced researchers in your field

- rapid publication on acceptance

- support for research data, including large and complex data types

- gold Open Access which fosters wider collaboration and increased citations

- maximum visibility for your research: over $100 \mathrm{M}$ website views per year

At $\mathrm{BMC}$, research is always in progress.

Learn more biomedcentral.com/submissions 